\title{
A Novel Neuron-Specific Regulator of the V-ATPase in Drosophila
}

\author{
Amina Dulac, ${ }^{1}$ A Abdul-Raouf Issa, ${ }^{1}$ Jun Sun, ${ }^{1}$ Giorgio Matassi, ${ }^{2,4}$ Célia Jonas, ${ }^{1}$ Zohra Rahmani, ${ }^{1}$ \\ Baya Chérif-Zahar, ${ }^{1}$ Daniel Cattaert, ${ }^{3}$ and ${ }^{D}$ Serge Birman ${ }^{1}$
}

https://doi.org/10.1523/ENEURO.0193-21.2021

\begin{abstract}
${ }^{1}$ Genes Circuits Rhythms and Neuropathology, Brain Plasticity Unit, Unité Mixte de Recherche 8249, Centre National de la Recherche Scientifique, École Supérieure de Physique et de Chimie Industrielles de la Ville de Paris, Paris Sciences et Lettres University, Paris F-75005, France, ${ }^{2}$ Dipartimento di Scienze Agroalimentari, Ambientali e Animali, University of Udine, Udine I-33100, Italy, ${ }^{3}$ Université de Bordeaux, Centre National de la Recherche Scientifique, Institut de Neurosciences Cognitives et Intégratives d'Aquitaine, Unité mixte de Recherche 5287, F-33000 Bordeaux, France, and ${ }^{4}$ Centre National de la Recherche Scientifique, Unité Mixte de Recherche 7058 "Ecologie et Dynamique des Systèmes Anthropisés" (EDYSAN), Université de Picardie Jules Verne, Amiens cedex F-80025, France
\end{abstract}

\begin{abstract}
The V-ATPase is a highly conserved enzymatic complex that ensures appropriate levels of organelle acidification in virtually all eukaryotic cells. While the general mechanisms of this proton pump have been well studied, little is known about the specific regulations of neuronal V-ATPase. Here, we studied CG31030, a previously uncharacterized Drosophila protein predicted from its sequence homology to be part of the V-ATPase family. In contrast to its ortholog ATP6AP1/VhaAC45 which is ubiquitous, we observed that CG31030 expression is apparently restricted to all neurons, and using CRISPR/Cas9-mediated gene tagging, that it is mainly addressed to synaptic terminals. In addition, we observed that CG31030 is essential for fly survival and that this protein co-immunoprecipitates with identified V-ATPase subunits, and in particular ATP6AP2. Using a genetically-encoded pH probe (VMAT-pHluorin) and electrophysiological recordings at the larval neuromuscular junction, we show that CG31030 knock-down induces a major defect in synaptic vesicle acidification and a decrease in quantal size, which is the amplitude of the postsynaptic response to the release of a single synaptic vesicle. These defects were associated with severe locomotor impairments. Overall, our data indicate that CG31030, which we renamed VhaAC45-related protein (VhaAC45RP), is a specific regulator of neuronal VATPase in Drosophila that is required for proper synaptic vesicle acidification and neurotransmitter release.
\end{abstract}

Key words: ATP6AP1L/ATP6AP1; CG31030/VhaAC45RP; Drosophila melanogaster; neuronal V-ATPase; quantal size; synaptic vesicle acidification

\section{Significance Statement}

In this study, we provide evidence that a previously uncharacterized Drosophila protein, CG31030, is necessary for fly survival and expressed specifically in neurons, where it interacts with constitutive and accessory subunits of the V-ATPase. Physiologically, we show that this protein is required for synaptic vesicle acidification and to ensure proper synaptic transmission at the neuromuscular junction. This implies that CG31030, alias VhaAC45RP, is a novel synaptic protein essential to nervous system functioning and neurotransmitter release. This work therefore provides a new step toward a more exhaustive understanding of the regulations of neuronal V-ATPase and their potential repercussions on synaptic transmission and neurologic diseases.

Received May 2, 2021; accepted September 30, 2021; First published October 7, 2021.

The authors declare no competing financial interests.
Author contributions: A.D., D.C., and S.B. designed research; A.D., A.-R.I., J.S., G.M., C.J., Z.R., B.C.-Z., D.C., and S.B. performed research and analyzed data; A.D. and S.B. wrote the paper with input from all authors. 


\section{Introduction}

Many cellular processes require a specific electrochemical environment for correct functioning, such as posttranslational modifications of proteins in the Golgi apparatus, lysosomal degradation, endosomal ligand-receptor dissociation, or hormone concentration (for review, see Forgac, 2007). Eukaryotic cells use a highly conserved proton pump, called the vacuolar $\mathrm{H}^{+}$-ATPase (V-ATPase), to achieve the adequate level of acidity in different cellular compartments (Saroussi and Nelson, 2009). This large enzymatic complex must be tightly regulated, as it is essential for it to be localized on the right membrane, and to fit the different $\mathrm{pH}$ ranges specific to each organelle and cell type.

In neurons, the V-ATPase plays a crucial role at the synapse, being responsible for acidifying synaptic vesicles and thus providing the driving force for neurotransmitter loading (Moriyama et al., 1992). Recently, neuronal VATPase has also gained interest in the context of aging and neurodegenerative diseases, as its dysregulation, and resulting impairment of the autophagy-lysosomal pathway, have been linked to several pathologies such as Alzheimer's and Parkinson's diseases (Colacurcio and Nixon, 2016; Collins and Forgac, 2020). If the core mechanism of the proton pump is now well understood, the regulations conferring the cell-specific functions of neuronal V-ATPase remain largely unknown, considerably limiting its potential use as a therapeutic target.

The V-ATPase complex is composed of a cytoplasmic domain $\left(V_{1}\right)$ and a membrane-bound domain $\left(V_{0}\right)$. The $V_{1}$ domain contains the catalytic unit responsible for ATP hydrolysis. The energy resulting from this reaction powers a rotational molecular motor spanning from $V_{1}$ to $V_{0}$, allowing protons to cross membranes through the port contained in $V_{0}$ (Vasanthakumar and Rubinstein, 2020). The assembly of $V_{1}$ to $V_{0}$ is necessary for the pump to function, and reversible dissociation of the two domains has been shown to occur as a way to regulate V-ATPase

This work was supported by the Fondation de France Grant Subvention 0086407 (to S.B.) and recurrent funding to S.B.'s laboratory from the École Supérieure de Physique et de Chimie Industrielles de la Ville de Paris and Centre National de la Recherche Scientifique. A.D., A.R.I., and J.S. were recipient of PhD fellowships from PSL University and Labex MemoLife, the Doctoral School 3C, and the China Scholarship Council, respectively.

A.-R. Issa's present address: Sussex Neuroscience, School of Life Sciences, University of Sussex, Brighton BN1 9QG, United Kingdom.

J. Sun's present address: Neurodevelopment Laboratory, School of Biosciences, University of Birmingham, Birmingham B15 2TT, United Kingdom.

Acknowledgements: We thank Dr. Philippe Marin for helpful advice about the quantitative analysis of mass spectrometry experiments, Dr. Hervé Tricoire and Dr. Elodie Martin for helpful suggestion on the CRISPR/Cas9 mutagenesis, Dr. Peter Robin Hiesinger for the gift of the anti-Vha100-1 antibody, Dr. David Krantz for providing the UAS-VMAT-pHluorin strain, Dr. Hermann Aberle for the OK371-Gal4 strain, Dr. Hervé Tricoire for the pCFD3U6:3-gRNA plasmid, and Dr. Céline Petitgas and Manon Dobrigna for participating in preliminary experiments during their student internships.

Correspondence should be addressed to Serge Birman at serge. birman@espci.fr.

https://doi.org/10.1523/ENEURO.0193-21.2021 Copyright (C) 2021 Dulac et al.

This is an open-access article distributed under the terms of the Creative Commons Attribution 4.0 International license, which permits unrestricted use, distribution and reproduction in any medium provided that the original work is properly attributed. activity (Collins and Forgac, 2020). Though the core mechanism stays the same, one V-ATPase can differ from another by its composition. In vertebrates, as well as in Drosophila, $V_{0}$ is made of five subunits $\left(a, c, c^{\prime \prime}, d\right.$, and $e$ ), while $V_{1}$ contains eight subunits (A-H; Allan et al., 2005). Each subunit can have several paralogs encoded by different genes, and each gene can produce several isoforms, allowing many different possible combinations to form the full V-ATPase complex. These differences of composition can also have regulatory effects on the complex, both on its localization and on its functional properties (Vasanthakumar and Rubinstein, 2020).

The V-ATPase can also be regulated by two accessory subunits, ATP6AP1/Ac45 and ATP6AP2/PRR (Jansen and Martens, 2012). Both proteins are found in the nervous tissue but are also required in other organs, and their mutations have been linked to cognitive impairments as well as systemic symptoms like immunodeficiency or hepatopathy (Jansen et al., 2016; Cannata Serio et al., 2018). These two subunits interact directly with the $\mathrm{V}_{0}$ domain and are believed to promote assembly of the membrane and soluble regions of the V-ATPase complex (Abbas et al., 2020). In addition, two homologs of ATP6AP1 have been described. The first one, called AC45-like protein (AC45LP), was identified in Xenopus where, unlike the ubiquitous AC45/ATP6AP1, it is expressed specifically in kidney and lung (Jansen et al., 2010). The second one, called ATP6AP1-like (ATP6AP1L) or Ac45-related protein (Ac45RP), was recently functionally characterized in mice where it also shows tissue-specificity, its expression being restricted to neurons (Jansen et al., 2021). These studies suggest that the AC45 family is larger than initially expected, and might play an important role in the tissue-specificity of the V-ATPase complex. Drosophila possesses identified orthologs of vertebrate ATP6AP1/Ac45 and ATP6AP2/PRR, named VhaAC45 and ATP6AP2, respectively. These proteins also seem to contribute to assembly of the V-ATPase in fly tissues (Schoonderwoert and Martens, 2002a; Guida et al., 2018).

In this study, we examined the localization and function of CG31030, a novel Drosophila ortholog of both ATP6AP1/Ac45 and ATP6AP1L/Ac45RP, whose characteristic is to be expressed selectively and ubiquitously in neurons. Whereas a complete deficiency of this protein is lethal, we found that partial CG31030 knock-down in larval motoneurons impaired synaptic vesicle acidification, reduced quantal size, which is the amplitude of the postsynaptic response to the release of a single synaptic vesicle, and induced severe locomotion defects. We also report that CG31030 from brain tissue co-immunoprecipitated with V-ATPase subunits of the $\mathrm{V}_{0}$ domain. Overall, our results indicate that CG31030 is a novel accessory subunit of the neuronal V-ATPase that appears to be involved in the regulation of synaptic activity.

\section{Materials and Methods}

\section{Drosophila culture and strains}

Flies were raised on standard agar-cornmeal-yeast medium, at $25^{\circ} \mathrm{C}$ in a $12 / 12 \mathrm{~h}$ light/dark cycle. CG31030 RNAi strains and mutants were obtained from the 
Table 1: Drosophila strains used in this study

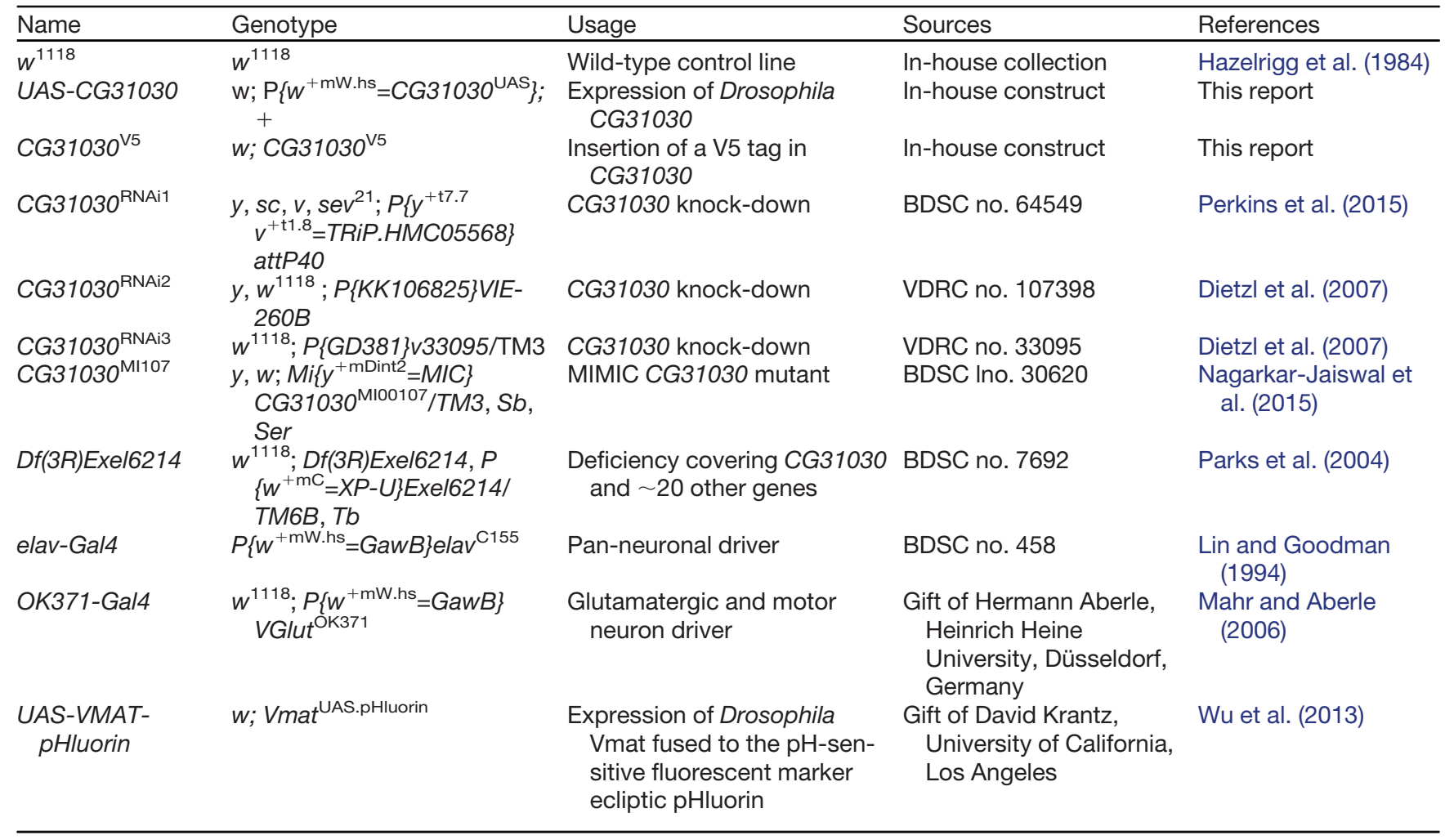

Bloomington Drosophila stock center (BDSC) and the Vienna Drosophila Resource Center (VDRC). Detailed genotypes and references of these lines are provided in Table 1. To construct the UAS-CG31030 strain, the CG31030 cDNA was PCR amplified from the clone RH09162 obtained from the Drosophila Genomics Resource Center, using the following primers with added restriction sites: P1EcoRI (forward) 5'-CCATCCGAATTCAAAATGCAGCTGATT CTCGT and P2-Xhol (reverse) 5'-TGGCTGCTCGAGATC TATTGGGTTATGAGAGA. The 1160-bp PCR fragment was inserted into pUAST (Brand and Perrimon, 1993), verified by sequencing (GATC Biotech) and sent to BestGene, for P-element transformation by random insertion in $w^{1118}$ background. A 2d-chromosome insertion of UAS-CG31030 that yielded strong expression of the transgene was used thereafter.

\section{Reverse transcription-coupled PCR and qPCR}

Total RNAs were extracted from 20 heads (or 15 thoraces or 15 abdomens) of 8-d-old flies using the QIAzol Lysis reagent (QIAGEN). The Maxima First Strand cDNA Synthesis kit (Thermo Fisher Scientific, K1671) was used with oligo(dT)20 primers to synthesize the cDNAs. Relative quantitative PCR assays were conducted using a LightCycler 480 and the SYBR Green I Master mix (Roche LifeScience), with Act5C as internal control for normalization of mRNA levels. All reactions were performed in triplicate. The specificity of amplification products was assessed by melting curve analyses. The following forward and reverse primers, were used: for CG31030, 5'-GGCTTCGTTGT
AGGCCAACAGA and 5'-CACCAGGTATCCCAAGTT CCAGA; for Act5C, 5'-CGTCGACCATGAAGATCAAG and 5'-TTGGAGATCCACATCTGCTG; for ATP6AP2, 5'-ACGATCCCTTCAACCTGGC and 5'-CCTGTCAGC TCGTAGGTCT; for VhaAC45, 5'-TGTCCCAAGTGG AGTTTGCC and 5'-TCAGGCCATTTTCCTCGAAGG; for Vha100-1, 5'-GGCATCCCCATGTTGGACA and 5'CGGCGTTCTGATTCACCTCC; for VhaAC39-1, 5'-GC AGGCTGACTATCTCAACCT and 5'-GCTCCACAGCAT GGTTCCTC; for Vha16-1, 5'-AAGTCTGGTACCGGTA TTGC and 5'-CCATGACCACAGGAATGATG. PCR assays were performed using the PrimeStar Max DNA polymerase (Takara) and the following primers: for CG31030-RA/RB, 5'- TGAGGTGTACGACTGCATAGGA and 5'- GAAAGAACTCGATGGCCAAGGT; for CG31030$R A$, 5'-CAAGGGATACGGTCGATTGAGCATCAC and 5'CTTACTCCTGTACGGTGAAGGTCAAC; for CG31030-RB, 5'- CAAGGGATACGGTCGATTGAGCATCAC and 5' ATGAACATGGCTGCCCGAGATTGTTTGCT.

\section{Protein extraction and Western blotting}

A total of 30 Drosophila heads were homogenized in 300- $\mu$ l RIPA buffer (Sigma-Aldrich) containing protease inhibitors (Roche Diagnostics, Complete Protease Inhibitor, Cocktail), using bead tubes and a Minilys apparatus (Bertin Technologies). Proteins samples and Western blotting were processed as previously described (Issa et al., 2018). Briefly, the extracted proteins were mixed with LDS sample buffer and reducing agent (Invitrogen, NuPAGE), heated at $70^{\circ} \mathrm{C}$ for $5 \mathrm{~min}$ and separated in 4-12\% Novex NuPAGE Bis-Tris precast polyacrylamide gels (Life Technologies) following the 
manufacturer's protocol in a MOPS-SDS running buffer. A semi-dry transfer was done onto polyvinylidene difluoride membranes (GE Healthcare Hybond P $0.45 \mu \mathrm{m}$ ) using a Hoefer TE77 apparatus. The mouse monoclonal antiV5 (Thermo Fisher Scientific, R960-25) was used, diluted at 1:500. Immunolabeled bands were revealed by ECL RevelBIOt Intense (Ozyme, OZYB002-1000) as chemiluminescent HRP substrate and digitally acquired using the ImageQuant TL software (GE Healthcare Life Science).

\section{CRISPR/Cas9 gene tagging}

The sequence of a V5 tag was inserted in frame after the coding sequence of the CG31030 gene, using a homology-directed repair CRISPR-Cas9 method (see scheme in Fig. 1D). The following guide RNA sequence: 5'TTCACCGTACAGGAGTAAGG-3' was cloned into the Bbsl site of pCFD3-U6:3-gRNA plasmid (Port et al., 2014; kind gift of Hervé Tricoire, Université de Paris, Paris, France). This plasmid was then injected, at a concentration of $500 \mathrm{ng} / \mu \mathrm{l}$, with the following single-stranded oligodeoxynucleotide (ssODN) donor repair template: 5'-GTTCGCG CAGCAAACAGTTGACCTTCACCGTACAGGAGTACGC AGGTAAGCCTATCCCTAACCCTCTCCTCGGTCTAGA TTCTACGTAAGGAGGTCATAAGTCTCTGATGAACCAATAGATCTCGGGC-3' (synthetized by Integrated DNA Technology), also at a concentration of $500 \mathrm{ng} / \mu \mathrm{l}$ (the sequence underlined corresponds to the in-frame V5 tag), into nos-cas9 embryos [genotype $y^{1}, P($ nos-cas9, $w+), M$ (3xP3-RFP.attP)ZH-2A, w*; Port et al., 2014]. An alanine was added before the V 5 tag to prevent the creation of a potential tyrosine phosphorylation site. Embryo injections were performed by BestGene. Single $F_{0}$ flies were crossed over the $T M 6 C(S b)$ balancer to establish stable lines. DNA was then extracted from three flies of each of these independent lines, and V5 insertion events were detected by dot blot using a mouse anti-V5 tag monoclonal antibody (Thermo Fisher Scientific, R96025). Positive strains were outcrossed in a $w^{1118}$ background and their genomic DNA was sequenced to check for proper in-frame V5 integration in CG31030. One of these equivalent CG31030 ${ }^{\mathrm{V} 5}$ mutant line was selected for further studies.

\section{Immunohistochemistry}

Adult brains of 8-d-old females, or third-instar larva CNS, were dissected in Drosophila Ringer's solution or hemolymph-like saline solution (HL3; $70 \mathrm{~mm} \mathrm{NaCl}, 5 \mathrm{~mm}$ $\mathrm{KCl}, 1.5 \mathrm{~mm} \mathrm{CaCl}_{2}, 70 \mathrm{~mm} \mathrm{MgCl}$, $10 \mathrm{~mm} \mathrm{NaHCO} 3,115$ $\mathrm{mm}$ sucrose, $5 \mathrm{~mm}$ trehalose, and $5 \mathrm{~mm}$ HEPES, with $\mathrm{pH}$ adjusted to 7.6), respectively, and fixed in $4 \%$ paraformaldehyde (Thermo Fischer Scientific) for $1 \mathrm{~h}$. After three 20 min washes in PBS plus $0.5 \%$ Triton X-100 (PBT), brains were blocked in PBT $+2 \%$ bovine serum albumin for $2 \mathrm{~h}$. They were then incubated in primary antibodies diluted in blocking solution for $24 \mathrm{~h}$ at $4^{\circ} \mathrm{C}$. The primary antibodies used were: mouse monoclonal anti-V5 (Thermo Fisher Scientific, R960-25, 1:200) and rat anti-Cadherin-N (CadN; DSHB, DN-Ex \#8, 1:20). Brains were then washed three times, for 20 min each, in PBT before being incubated in secondary antibodies for $2 \mathrm{~h}$. Secondary antibodies used were: Alexa Fluor 488 anti-mouse and Alexa Fluor 555 antirat (Fisher Scientific, A11029 and A21434, respectively), all diluted at 1:1000. After two 20-min washes in PBT followed by two 20-min washes in PBS, brains were mounted in Prolong Gold Antifade Mountant (Thermo Fisher Scientific, P36930). Imaged were acquired on a Nikon A1R confocal microscope.

For immunostaining of the larval muscles and neuromuscular junctions, the Alexa Fluor 488 Tyramide SuperBoost kit (Thermo Fisher Scientific, B40912) was used to increase the V5 signal that was otherwise faint in this tissue. The working protocol was as recommended by the manufacturer, with anti-V 5 diluted to $1: 100$, then followed by classical immunostaining, as described above, to co-stain for the nerve terminals with an anti-HRP antibody (Jackson ImmunoResearch, 323-005-021, 1:200) and an anti-Vha100-1 (courtesy of Dr. Peter Robin Hiesinger, 1:2000).

\section{Longevity assay}

Approximately 110 virgin females from each genotype were collected on their hatching day and placed in clean bottles, with no more than 25 flies per bottle. Flies were transferred in new clean bottles, and survivors were counted, every $2 \mathrm{~d}$ for $60 \mathrm{~d}$.

\section{Co-immunoprecipitation coupled to nano liquid chromatography coupled to tandem mass spectrometry (LC-MS/MS) analysis}

Approximately 200 heads from 8-d-old CG $1030^{\mathrm{V} 5}$ and $w^{1118}$ flies were lysed using glass beads in $500 \mu \mathrm{l}$ of icecold lysis buffer: 50 mm Tris-HCl pH 7.4, 2 mm EDTA, 150 $\mathrm{mm} \mathrm{NaCl}, 0.5 \%(\mathrm{v} / \mathrm{v})$ IGEPAL CA-630 (Sigma-Aldrich, I3021), 10\% (v/v) glycerol, 1 mм PMSF protease inhibitor (Sigma-Aldrich, P-7626), and $1 \times$ cOmplete Mini Protease Inhibitor Cocktail (Roche, 11836153001). Samples were left on ice, with occasional gentle agitation, for $30 \mathrm{~min}$ before being centrifuged at $12,000 \mathrm{rpm}(13,000 \times \mathrm{g})$ at $4^{\circ} \mathrm{C}$ for $10 \mathrm{~min}$ to remove insoluble material; $400 \mu \mathrm{l}$ of the supernatants were then added to $50 \mu$ l of Anti-V5-tag mAb-Magnetic Beads (MBL International, M167-11), that had been previously washed as recommended by the manufacturer. Samples were incubated with gentle agitation at $4^{\circ} \mathrm{C}$ for $4 \mathrm{~h}$. The supernatants were removed using a magnetic rack, and beads were washed three times with $500 \mu \mathrm{m}$ ice-cold lysis buffer before being resuspended in $10 \mu \mathrm{l}$ of milliQ water.

Nano LC-MS/MS was performed at the Proteomics facility of the Institut Jacques Monod (Université de Paris). Proteins on beads were digested overnight at $37^{\circ} \mathrm{C}$ with trypsin (Promega) in $25 \mathrm{~mm} \mathrm{NH}_{4} \mathrm{HCO}_{3}$ buffer $(0.2 \mu \mathrm{g}$ trypsin in $20 \mu \mathrm{l})$. The resulting peptides were desalted using ZipTip $\mu$-C18 Pipette Tips (Pierce Biotechnology). Eluates were analyzed using either an Orbitrap Fusion or an Orbitrap Q-Exactive Plus, coupled, respectively, to a Nano-LC Proxeon 1200 or a Nano-LC Proxeon 1000, both equipped with an easy spray ion source (Thermo Fisher Scientific). 
On the Orbitrap Fusion instrument, peptides were loaded with an online preconcentration method and separated by chromatography using a Pepmap-RSLC C18 column $(0.75 \times 750 \mathrm{~mm}, 2 \mu \mathrm{m}, 100 \AA)$ from Thermo Fisher Scientific, equilibrated at $50^{\circ} \mathrm{C}$ and operated at a flow rate of $300 \mathrm{nl} / \mathrm{min}$. Peptides were eluted by a gradient of solvent $A\left(\mathrm{H}_{2} \mathrm{O}, 0.1 \% \mathrm{FA}\right)$ and solvent $\mathrm{B}\left(\mathrm{ACN} / \mathrm{H}_{2} \mathrm{O} 80 / 20\right.$, $0.1 \% \mathrm{FA}$ ). The column was first equilibrated for $5 \mathrm{~min}$ with $95 \%$ of $A$, then $B$ was raised to $28 \%$ in 105 min and to $40 \%$ in 15 min. Finally, the column was washed with $95 \%$ B during $20 \mathrm{~min}$ and re-equilibrated at $95 \%$ A for $10 \mathrm{~min}$. Peptides masses were analyzed in the Orbitrap cell in full ion scan mode, at a resolution of 120,000 , a mass range of $\mathrm{m} / \mathrm{z} 350-1550$ and an AGC target of $4.10^{5}$. MS/MS were performed in the top speed $3 \mathrm{~s}$ mode. Peptides were selected for fragmentation by higher-energy C-trap dissociation (HCD) with a normalized collisional energy of $27 \%$ and a dynamic exclusion of $60 \mathrm{~s}$. Fragment masses were measured in an lon trap in the rapid mode, with and an AGC target of $1.10^{4}$. Monocharged peptides and unassigned charge states were excluded from the MS/MS acquisition. The maximum ion accumulation times were set to $100 \mathrm{~ms}$ for MS and $35 \mathrm{~ms}$ for MS/MS acquisitions, respectively.

On the Q-Exactive Plus instrument, peptides were loaded with an online preconcentration method and separated by chromatography using a Pepmap-RSLC C18 column $(0.75 \times 500 \mathrm{~mm}, 2 \mu \mathrm{m}, 100 \AA$ A $)$ from Thermo Scientific, equilibrated at $50^{\circ} \mathrm{C}$ and operated at a flow rate of $300 \mathrm{nl} / \mathrm{min}$. Peptides were eluted by a gradient of solvent $A\left(\mathrm{H}_{2} \mathrm{O}, 0.1 \% \mathrm{FA}\right)$ and solvent $B(100 \% A C N$, $0.1 \% \mathrm{FA}$ ), the column was first equilibrated $5 \mathrm{~min}$ with $95 \%$ of $A$, then $B$ was raised to $35 \%$ in 93 min and finally, the column was washed with $80 \%$ B during $10 \mathrm{~min}$ and re-equilibrated at $95 \%$ A during $10 \mathrm{~min}$. Peptides masses were analyzed in the Orbitrap cell in full ion scan mode at a resolution of 70,000 with a mass range of $\mathrm{m} / \mathrm{z} \quad 375-1500$ and an AGC target of $3.10^{6}$. MS/MS were performed in a Top 20 DDA mode. Peptides were selected for fragmentation by HCD with a normalized collisional energy of $27 \%$, and a dynamic exclusion of $30 \mathrm{~s}$. Fragment masses were measured in the Orbitrap cell at a resolution of 17,500 , with an AGC target of $2.10^{5}$. Monocharged peptides and unassigned charge states were excluded from the MS/MS acquisition. The maximum ion accumulation times were set to $50 \mathrm{~ms}$ for MS and $45 \mathrm{~ms}$ for MS/MS acquisitions, respectively.

Raw data were processed on Proteome Discoverer 2.4 with the mascot node (Mascot version 2.5.1) against the Swissprot/TrEMBL protein database release 2019_12 for Drosophila melanogaster. A maximum of two missed cleavages was authorized. Precursor and fragment mass tolerances were set to, respectively, $7 \mathrm{ppm}$ and $0.5 \mathrm{Da}$ (Orbitrap Fusion) and to $6 \mathrm{ppm}$ and 0.02 Da (Orbitrap Qexactive Plus). The following posttranslational modifications were included as variable: acetyl (protein N-term), oxidation (M), phosphorylation (STY). Peptide identifications were validated with a 1\% FDR (false discovery rate) threshold calculated with the Percolator algorithm. Label- free quantification was done in TOP three abundance calculation mode with pairwise ratio-based calculation and $t$ test (background based) hypothesis test. Only proteins identified in at least one group in two independent experiments were kept in the analysis. Missing values were set to the minimum abundance of the experiment.

\section{VMAT-pHluorin experiments}

Third-instar larvae expressing VMAT-pHluorin with or without CG31030 RNAi in motor neurons using the OK371-Gal4 driver, were dissected to expose the body wall muscles in $\mathrm{Ca}^{2+}$-free $\mathrm{HL} 3$ saline solution $(70 \mathrm{~mm}$ $\mathrm{NaCl}, 5 \mathrm{~mm} \mathrm{KCl}, 70 \mathrm{~mm} \mathrm{MgCl} 2,10 \mathrm{~mm} \mathrm{NaHCO}$, $115 \mathrm{~mm}$ sucrose, $5 \mathrm{~mm}$ trehalose, and $5 \mathrm{~mm}$ HEPES, pH 7.6). Two other solutions were used: an acidic $\mathrm{Ca}^{2+}$-free $\mathrm{HL} 3$ saline $\left(\mathrm{pH}\right.$ 5.5) and a neutral $\mathrm{HL} 3-\mathrm{NH}_{4} \mathrm{Cl}$ saline $(\mathrm{pH} 7.6)$, in which $50 \mathrm{~mm} \mathrm{NaCl}$ was replaced by $50 \mathrm{~mm} \mathrm{NH}_{4} \mathrm{Cl}$. After dissection, larvae fillets were allowed to settle in $\mathrm{Ca}^{2+}$-free $\mathrm{HL} 3$ saline for $10 \mathrm{~min}$ before being scanned a first time directly in a drop of the solution using a Nikon A1R confocal microscope. $\mathrm{Ca}^{2+}$-free HL3 saline was then replaced either by the acidic $\mathrm{Ca}^{2+}$-free $\mathrm{HL} 3$ saline for low $\mathrm{pH}$-induced quenching experiments, or by the $\mathrm{HL} 3-\mathrm{NH}_{4} \mathrm{Cl}$ saline for $\mathrm{pH}$ gradient collapse experiments. In both cases, larval fillets were rinsed three times with the modified solution, and incubated for $3 \mathrm{~min}$, before being scanned a second time, in a drop of modified solution. Quantification was done on Z-projections (set to maximal intensity) of confocal stacks. Using the Fiji software, a fixed threshold was applied to all images to get rid of the background and select only synaptic areas. Percentage of area over the threshold was used as a measure of the signal intensity and ratios of the values obtained in modified solutions over standard HL3, i.e., the second scan over the first scan, were calculated for quantifications.

\section{Larval locomotion assays}

Larval locomotion was assessed on an in-house made version of the FIMtable system (Risse et al., 2014). Thirdinstar larvae were collected and briefly rinsed in water to remove traces of food, before being gently placed on the recording table precoated with a thin layer of $1.2 \%$ agar gel. Only four larvae of the same genotype were recorded simultaneously, to avoid collisions between animals, for a period of 2 min with a Basler ace acA2040-25gm camera at $12.5 \mathrm{frames} / \mathrm{s}$. Larvae that burrowed themselves into the agar plate or escaped the arena before the end of recording were excluded from the results. Tracking was done using the FIMtrack software, as described in (Risse et al., 2014). The number of peristaltic waves was computed from the variations in larval area. More precisely, the curves of area variation were first smoothed with a Savitzky-Golay filter to get rid of unwanted noise, then the number of waves was defined as the number of peaks on the curve (which was automatically computed by a custom-made Python script). Stride size was then calculated as the distance traveled by the larva divided by the number of peristaltic waves, while stride duration was 
defined as the recording time (i.e., 120 s) divided by the number of peristaltic waves.

\section{Electrophysiological recordings and quantal analysis}

Third-instar larvae expressing CG31030 RNAi in motor neurons using the OK371-Gal4 driver, and appropriate controls, were dissected to expose the body wall muscles, and the brain removed, in $\mathrm{Ca}^{2+}$-free $\mathrm{HL} 3$ saline solution $(70 \mathrm{~mm}$ $\mathrm{NaCl}, 5 \mathrm{~mm} \mathrm{KCl}, 70 \mathrm{~mm} \mathrm{MgCl} 2,10 \mathrm{~mm} \mathrm{NaHCO} 3,115 \mathrm{~mm}$ sucrose, $5 \mathrm{~mm}$ trehalose, and $5 \mathrm{~mm}$ HEPES, pH 7.6; Stewart et al., 1994; Cattaert and Birman, 2001). Spontaneous miniature EPSPs (mEPSPs) were recorded in the presence of tetrodotoxin (TTX) $10^{-6} \mathrm{M}$, so that no spike could occur, from the ventral longitudinal abdominal muscle 6 in segment A3.

Quantal analysis was performed following the theoretical background described in (Kuno, 1971) and (Castellucci and Kandel, 1974). Distribution histograms of mEPSP size were built from each muscle fiber recording with a $0.01-\mathrm{mV}$ bin size. These histograms provided an estimate of the mean size of a unitary EPSP, since the peaks represent integer multiples of the unitary size. A theoretical distribution was then computed by convolving a binomial distribution, accounting for quantal content (number of quanta released), and a Gaussian distribution, allowing for variations in size of individual quanta. More precisely, the probability for an EPSP to contain $i$ quanta follows a binomial distribution:

$$
\mathrm{P}(i)=\mathrm{C}_{n}^{i} \times p^{i} \times(1-p)^{(n-i)},
$$

in which $p$ is the average probability of release, and $n$ is the total number of releasable quanta. In the frequency distribution of EPSP amplitude, successive peaks represent increasing numbers of quanta. In order to predict how these events are distributed between different bins in a histogram, it is necessary to allow for variations in quantal size. To do this, the largest peaks of the histogram were fitted to a Gaussian curve scaled in width to have a variance proportional to quantal size, and scaled in height so that its area corresponded to the predicted number of events (Del Castillo and Katz, 1954). From the mean $(\mu)$ and SD $(\sigma)$ of the Gaussian curve, the content $(f)$ of each bin $(y)$ is given by:

$$
f(y)=\frac{1}{\sqrt{2 \pi} \sigma} e^{-\frac{1}{2}\left(\frac{y-\mu}{\sigma}\right)^{2}} .
$$

The SD $(\sigma)$ of each peak depends on the number of quanta it contains. It was calculated as follows:

$$
\sigma=i \times \sqrt{\sigma_{0}},
$$

with $i$ the number of quanta in the peak, and $\sigma_{0}$ the SD of a single quanta. The amplitude of Gaussian distribution for each peak is scaled to the probability $P(i)$ for that peak (see Eq. 1). The complete theoretical distribution, allowing for variance and peak overlap was then obtained by pooling the Gaussians for all peaks. In this way, the theoretical distribution could be superimposed on the histogram for direct comparison with the data.

\section{Endocytosis and exocytosis measurements}

We used fluorescent FM dye labeling to monitor synaptic vesicle cycling at the larval neuromuscular junction (Verstreken et al., 2008). Third-instar larvae expressing mCD8::GFP with or without CG31030 RNAi in motor neurons under control of the OK371-Gal4 driver, were dissected in a $\mathrm{Ca}^{2+}$-free HL3 saline solution (as described above), and incubated for $5 \mathrm{~min}$ in HL3 solution supplemented with $90 \mathrm{mM} \mathrm{KCl}, 1.5 \mathrm{~mm} \mathrm{CaCl}_{2}$ and $10 \mu \mathrm{M}$ fluorescent FM4-64 dye (Invitrogen, T13320) final concentrations. Preparations were then washed with $\mathrm{Ca}^{2+}$ free HL3 saline five times, for 2 min each, before being imaged on a Nikon A1R confocal microscope to measure the level of dye loading. Then the fillets were incubated for 2 min in HL3 solution supplemented with 90 $\mathrm{mm} \mathrm{KCl}$ and $1.5 \mathrm{~mm} \mathrm{CaCl}_{2}$ to allow the dye to be unloaded from the synapses. Preparations were then washed with $\mathrm{Ca}^{2+}$-free $\mathrm{HL} 3$ saline five times, for $2 \mathrm{~min}$ each, before being scanned once again to measure the level of dye unloading. Images were analyzed using the Fiji software. The fluorescence signal of the dye was measured in the synaptic area restricted to the signal of mCD8::GFP. To obtain the percentage of dye that was unloaded, the ratio of unloaded/loaded fluorescence intensities was calculated.

\section{Synapse morphology analysis}

Immunostaining was performed as described above in third-instar larvae using an antibody against Discs Large (Dlg; DSHB, catalog \#4F3 anti-discs large-s, 1:500). Synaptic boutons were then counted manually on the ventral longitudinal abdominal muscle 6 of the segment $A 3$, and their diameter measured in blind on randomized images using the Fiji software.

\section{Statistical treatment}

Statistical analysis was performed with the GraphPad Prism 6 software. The paired Student's $t$ test was used for comparison of two genotypes, while either paired or unpaired ANOVA, with Dunnett's post hoc test for multiple comparisons, were used for three genotypes.

\section{Results}

\section{Drosophila CG31030 encodes a single protein structurally similar to VhaAC45}

Here, we studied the Drosophila gene CG31030, identified in FlyBase as a paralog of CG8029/VhaAC45, and as an ortholog of vertebrate ATP6AP1/AC45 and ATP6AP1L/ AC45RP (Thurmond et al., 2019). In FlyBase, CG31030 is predicted to produce two different protein isoforms, CG31030-PA and CG31030-PB, which differ in the sequence of their $\mathrm{C}$-terminal domain, the last $7 \mathrm{C}$-terminal amino-acids of CG31030-PA being theoretically replaced by a different segment of 46 amino-acids in CG31030-PB. We first investigated the existence of these two isoforms. Using primers framing the $55 \mathrm{bp}$ difference between the predicted CG31030-RA and CG31030-RB transcripts (Fig. 1A), which encode CG31030PA and CG31030-PB, respectively, we checked which 
A

CG31030-RA

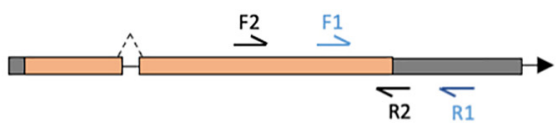

CG31030-RB

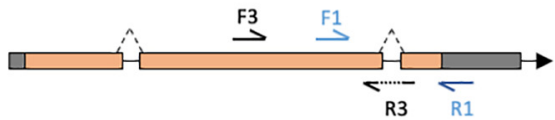

C

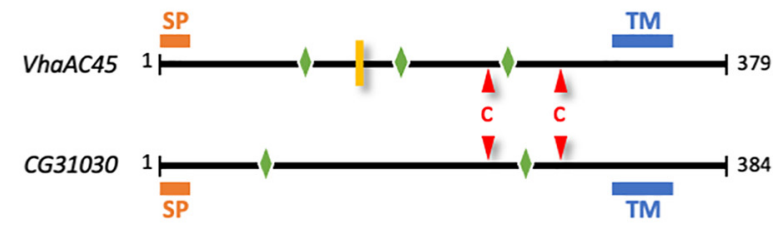

E

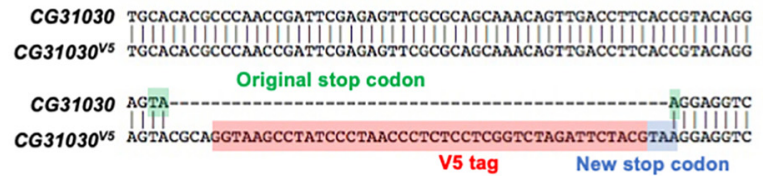

B

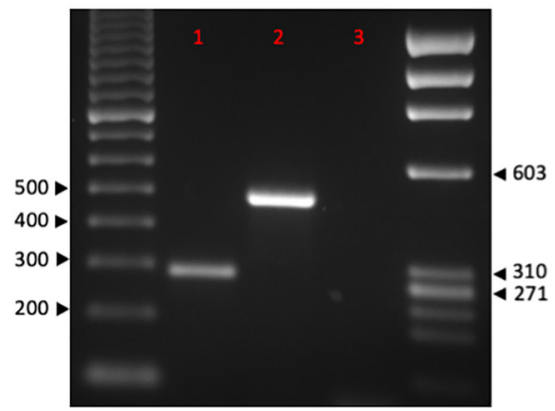

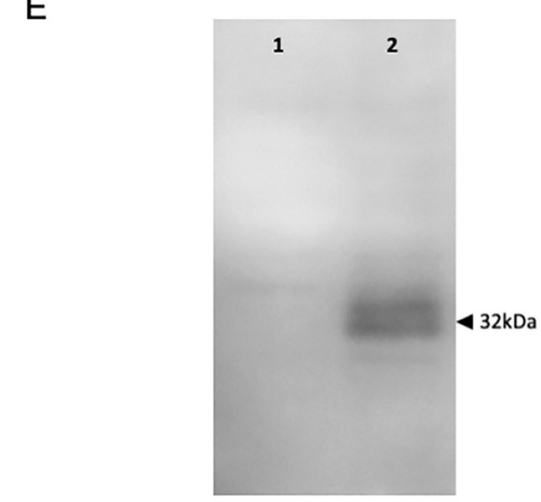

Figure 1. Structure of CG31030 compared with its paralog VhaAC45 and construction of CG31030 ${ }^{\mathrm{V}}$. $\boldsymbol{A}$, Localization of the primers used for RT-PCR analysis of the two predicted CG31030 transcripts (CG31030-RA and CG31030-RB) in Drosophila heads. B, Primers detecting both CG31030-RA (F1-R1, expected amplicon size $288 \mathrm{bp}$ ) and CG31030-RB (F1-R1, expected amplicon size $233 \mathrm{bp}$ ) only amplify a band corresponding to CG31030-RA (lane 1). CG31030-RA-specific primers (F2-R2) also amplify a fragment of the expected size (485 bp, lane 2), whereas CG31030-RB-specific primers (F3-R3) did not amplify any cDNA fragment (lane 3). C, VhaAC45 and CG31030 both have a signal peptide (SP; orange bars), a transmembrane domain (TM; blue bars) and predicted Nglycosylation sites according to NetNGlyc (http://www.cbs.dtu.dk/services/NetNGlyc/; green diamonds). They also present the pair of cysteine residues characteristic of the AC45 family (red triangles). In addition, VhaAC45 has a predicted furin cleavage site (yellow bar) which is absent in CG31030 according to ProP 1.0 (http://www.cbs.dtu.dk/services/ProP/). D, Construction of the CG31030 5 mutant strain. A 14-amino acid V5 tag (in red) was fused to the C-terminal end of the CG31030 protein by inserting the V5 coding sequence ended by a new stop codon (in blue) in place of the original stop codon (in green) in the CG31030 gene using the CRISPR-Cas9 technology. $\boldsymbol{E}$, A Western blotting probed with anti-V5 antibody detected two protein bands at an apparent molecular weight of $\sim 32 \mathrm{kDa}$ in head extracts of CG31030 $\mathrm{V5}$ flies (lane 2) and none in the $w^{1118}$ control sample (lane 1).

isoform was present in Drosophila heads. Only a fragment with the size expected for CG31030-RA was amplified (Fig. 1B), suggesting that CG31030-PB is not expressed in this tissue. Consistent with this, PCR experiments using CG31030-RB-specific primers did not amplify any cDNA fragment, contrary to experiments using CG31030-RA-specific primers (Fig. 1B). Moreover, among the numerous cDNA clones matching CG31030 listed in FlyBase, either fully sequenced or only sequenced at the end (ESTs), most of which complement both isoforms, several were found to be specific to CG31030-RA. In contrast, none specifically corresponded to $C G 31030-R B$, supporting the hypothesis that among the two predicted protein isoforms, only CG31030-PA is actually expressed in Drosophila. Therefore, we referred to CG31030-PA simply as CG31030 in the present study.
CG31030 and VhaAC45 share $69.9 \%$ similarity in amino-acid sequences. Like its paralog, CG31030 is also classified in the V-ATPase family group by the InterPro database (accession Q8IMJ0_DROME; Mitchell et al., 2019). They both have a transmembrane domain, as well as predicted $\mathrm{N}$-glycosylation sites (Fig. 1C). They also present a pair of conserved cysteine residues typical of proteins from the AC45 family. Unlike Drosophila VhaAC45 and vertebrate ATP6AP1/AC45, CG31030 does not possess a potential furin cleavage site (Fig. 1C), similarly to vertebrate ATP6AP1L/AC45RP and Xenopus AC45LP (Jansen et al., 2010, 2021).

Using the CRISPR-Cas9 technology, we inserted a small V5 epitope tag in frame at the $3^{\prime}$ end of the CG31030 gene, thus disrupting the stop codon, to generate the CG31030 ${ }^{\mathrm{V}}$ mutant line (Fig. 1D). A Western blotting on head extracts of CG31030 ${ }^{\mathrm{V} 5}$ and control flies was then performed to 
A

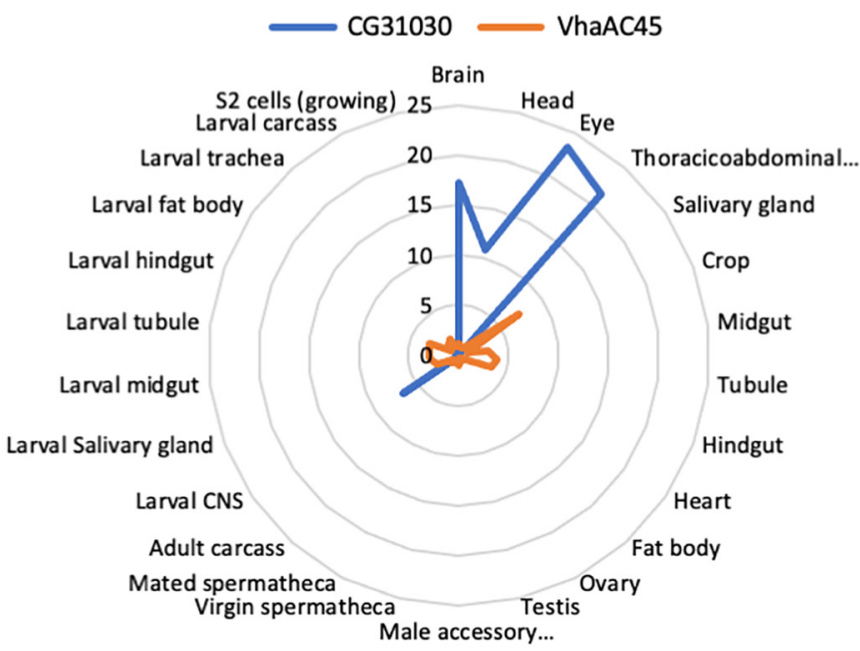

C

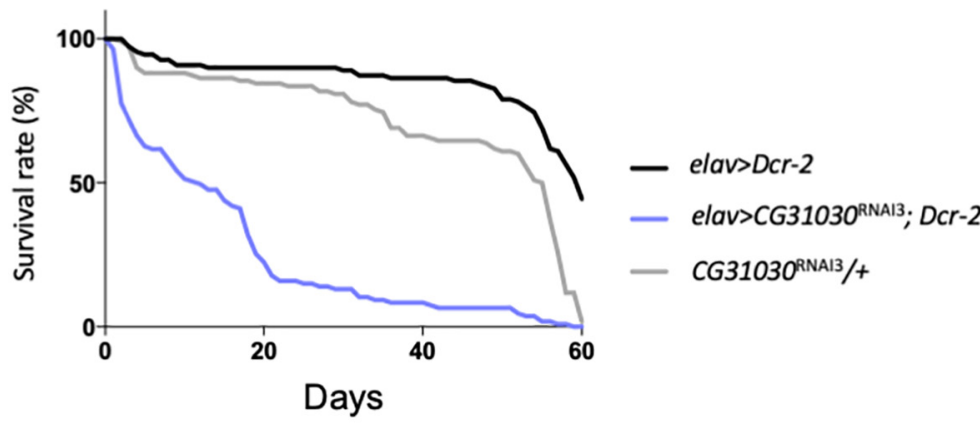

B

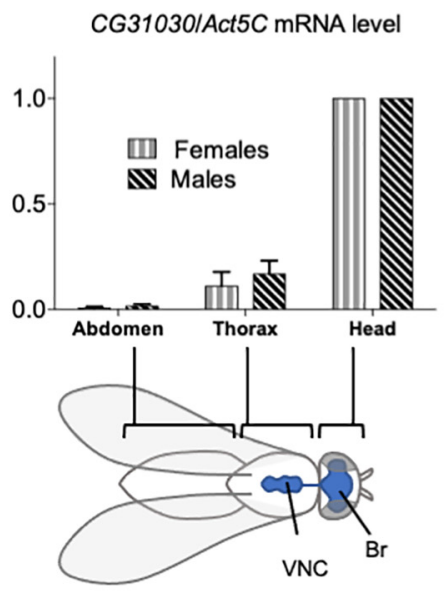

D

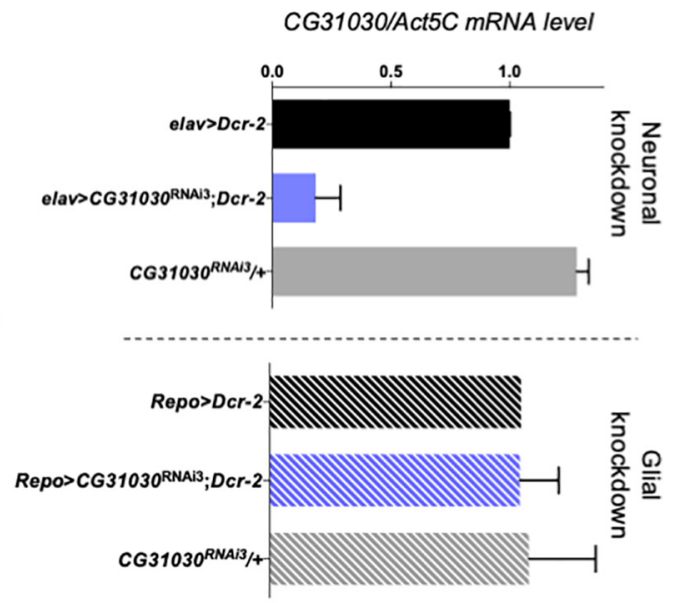

Figure 2. CG31030 is expressed specifically in neurons. A, Diagram representing expression levels of CG31030 and VhaAC45 in different tissues relative to the whole fly, according to FlyAtlas data (Chintapalli et al., 2007). CG31030 appears to be markedly enriched in the nervous system of larva and adult fly, whereas in contrast VhaAC45 seems to be uniformly expressed in all tissues in these two stages. $\boldsymbol{B}$, In both males and females, CG31030 mRNA abundance follows the localization of the CNS (shown in blue on the fly sketch), with the highest expression in the head. Br, brain; VNC, ventral nerve cord. Results of three independent experiments. C, Knock-down of CG31030 expression in neurons decreases adult longevity. Pan-neuronal expression of CG31030 ${ }^{\mathrm{RNAi}}$ and $\mathrm{Dcr}-2$ with the elav-Gal4 driver led to a marked shortening of the lifespan of adult flies. Experiment conducted with 105-110 females per genotype. D, Expression of CG31030 ${ }^{\mathrm{RNAi}} 3$ with Dcr-2 in all neurons using elav-Gal4 decreased CG31030 mRNA level in head by $>80 \%$, while expression of the RNAi construct and Dcr-2 in all adult glial cells with repo-Gal4 had not effect. Results of three independent experiments. Mean values with SD are reported on the graphs.

determine the apparent size of the tagged protein. Two distinct bands, close to each other, were detected specifically in the CG31030 ${ }^{\mathrm{V} 5}$ sample, consistent with the presence of glycosylated and unglycosylated forms of the same protein (Fig. $1 E$ ). While the predicted size of CG31030-V5 is $44 \mathrm{kDa}$, the observed bands corresponded to a size of $\sim 32 \mathrm{kDa}$. This could indicate that the protein is actually cleaved, like its paralog VhaAC45, despite the absence of a predicted cleavage site (Fig. 1C). However, it is known that membrane proteins migrate faster on SDS gels, leading to an underestimation of their size (Rath et al., 2009). This could also explain the smaller than expected apparent size of CG31030-V5 on Western blotting.

\section{CG31030 is specifically expressed in neurons and addressed to synaptic areas}

According to FlyAtlas (Chintapalli et al., 2007), VhaAC45 is expressed ubiquitously in Drosophila tissues. In contrast and interestingly, CG31030 seems to be specifically expressed in the nervous system in both larval and adult flies, making it a possible candidate for specific regulation of neuronal V-ATPase (Fig. 2A). To confirm this prediction, we checked by RT-qPCR the repartition of CG31030 transcripts in three parts of the fly body: the head and thorax, which contains the brain and ventral nerve cord (VNC), respectively, and the abdomen, which is relatively poor in nervous 
Table 2: Pan-neuronal expression of CG31030 rescues the embryonic lethality of CG31030-deficient flies up to the adult stage

\begin{tabular}{lcc}
\hline Genotype of the progeny flies & & Scored percentage $^{3}$ \\
\hline Heterozygous deficiency without CG31030 expression & Expentage $^{2}$ & 39.7 \\
Heterozygous deficiency with pan-neuronal CG31030 expression & 40 & 53.8 \\
Homozygous deficiency without CG31030 expression & 0 & 0 \\
Homozygous deficiency with pan-neuronal CG31030 expression & 20 & 6.4
\end{tabular}

\footnotetext{
${ }^{1}$ Rescue of embryonic lethality was assayed by crossing elav-Gal4; CG31030 Ml107/TM6B(Tb) females with w; UAS-CG31030/CyO; Df(3R)Exe/6214/TM6B(Tb) males, and scoring the relative number of CG31030-expressing adult flies homozygous for CG31030 deficiency (i.e., non-Tb and non-Cy elav-Gal4/+; UASCG31030/+; CG31030 $\mathrm{Ml107} / \mathrm{Df}(3 R)$ Exe/6214) in the progeny (highlighted line).

${ }^{2}$ Expected percentage of adult progeny flies of each genotype in case of full rescue.

${ }^{3}$ Actual percentage obtained in the experiments. 24 rescued adults were recovered out of a total of 373 progeny flies.
}

tissue. Both females and males showed highest expression in the head, minor expression in the thorax and no detectable expression in the abdomen (Fig. 2B). The expression of CG31030 therefore closely follows the repartition of the nervous system in adult flies.

The single-cell RNA-Seq Scope database (Davie et al., 2018) furthermore indicated that, in Drosophila, CG31030 is expressed in all neurons, with few or no expression in glial cells. To verify this, we expressed three different CG31030 RNAi with either the pan-neuronal driver elavGal4 or the pan-glial driver repo-Gal4 (see Table 1 for genotypes of the different Drosophila lines used in this study). The expression of CG31030 RNAi1 and CG31030 RNAi2 was found to be lethal at embryonic and first larval stages, respectively, while CG31030 RNAi3 produced viable adults with a shortened lifespan (Fig. 2C), and obvious locomotor impairments. This difference in phenotypes observed with different RNAi constructs could be attributed to a variation in residual levels of the CG31030 protein. RT-qPCR experiments showed that the pan-neuronal expression of CG31030 ${ }^{\mathrm{RNAi} 3}$ together with the RNAi booster Dicer-2 (Dcr-2) was sufficient to decrease by $>80 \%$ CG31030 transcripts abundance in extracts from the adult heads (Fig. 2D). On the other hand, glial expression of this RNAi construct with repo-gal4 had no significant effects on CG31030 transcript level (Fig. 2D), confirming that this gene is selectively expressed in neurons. It is interesting to note that both CG31030 ${ }^{\mathrm{RNAi} 1}$ and CG31030 ${ }^{\mathrm{RNA} 2}$ also induced lethal phenotypes at various developmental stages when expressed either with a glutamatergic (VGlut-Gal4) or a cholinergic (Cha-Gal4) neuronal driver (data not shown), in accordance with the RNA-Seq data of the Scope website suggesting a pan-neuronal expression of this gene.

To validate these observations, we placed the available MIMIC allele CG31030 ${ }^{\mathrm{MI} 107}$ that contains a stop codon inserted in the middle of the gene (Nagarkar-Jaiswal et al., 2015) over the deficiency Df(3R)Exel6214 encompassing CG31030 (Parks et al., 2004). The resulting mutant, likely to be a null, was found to be embryonic lethal, in agreement with the results obtained with two CG31030 RNAi lines. Remarkably, re-expressing the gene selectively in neurons, using a UAS-CG31030 construct driven by elavGal4, was sufficient to rescue this lethality, producing viable and fertile adults with no obvious behavioral defects (Table 2). These results convincingly indicate that CG31030 is an essential gene whose expression appears to be specifically required in neurons.
Next, we studied the cellular localization of this protein by performing immunostaining on CG31030 ${ }^{\mathrm{V} 5}$ flies with an anti-V5 tag antibody. We observed that the general expression pattern of CG31030 in adult brain (Fig. 3A) and larval CNS (Fig. 3B) was widespread in the neuropile and quite similar to that of the synaptic marker CadN, consistent with a predominantly synaptic localization. No specific signal was detected in a control $w^{1118}$ line that does not contain the V5-tagged protein (Fig. 3C). Neuronal cell bodies were also faintly marked with the V5 antibody in the CG31030 V5 line, both in adult and larva, and a few of them displayed a signal intensity comparable to that of the synaptic areas (Fig. $3 A, B$, arrowheads). The synapsecontaining neuropile areas (like the antennal lobes) were not as sharply defined as with the CadN antibody, suggesting that axons, like most cell bodies, also contains low levels of CG31030. Finally, co-immunostaining of larval body muscles wall with anti-horseradish peroxidase (HRP) antibodies, a marker of Drosophila neurons (Jan and Jan, 1982), showed precise co-localization with the V5 signal, indicating that CG31030 is addressed to synaptic boutons at the larval neuromuscular junction (Fig. 3D). The lack of V5 immunostaining at the neuromuscular junction of control $w^{1118}$ flies is shown below in Figure 4D.

\section{CG31030 co-immunoprecipitates with V-ATPase subunits of the $\mathbf{V}_{\mathbf{0}}$ domain}

The CG31030 protein is predicted to be part of the InterPro V-ATPase family, but no experimental information is currently available regarding its potential interactors. To determine whether CG31030 could interact, directly or indirectly, with subunits of the V-ATPase complex, we conducted co-immunoprecipitation experiments using a V5 antibody on proteins extracted from heads of CG31030 V5 mutants and $w^{1118}$ control flies, followed by nano LC-MS/MS analysis of the precipitated proteins. Three independent experiments were performed to increase reliability, and in total, 410 proteins were identified in all three experiments. Among those, only 12 proteins had at least a 2-fold abundance difference with the control in all three experiments (Fig. 4A; Table 3), one of them being, as expected, the co-immunoprecipitation target CG31030-V5. Remarkably, three of the other proteins were identified as subunits of the V-ATPase complex: Vha100-1, VhaAC39-1, and ATP6AP2 (Fig. 4B). 
A

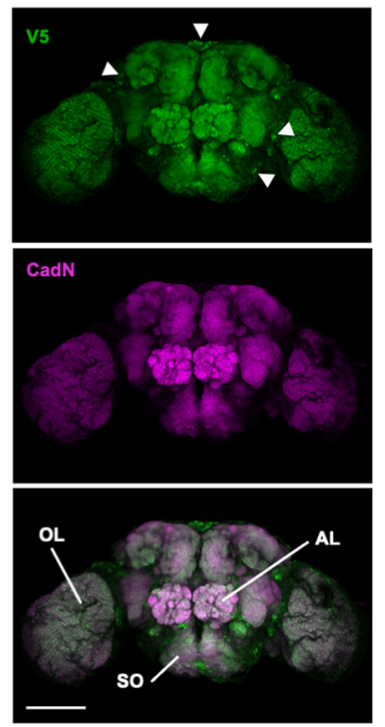

D

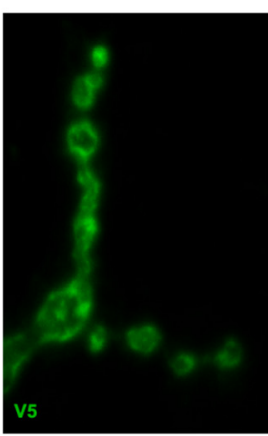

B
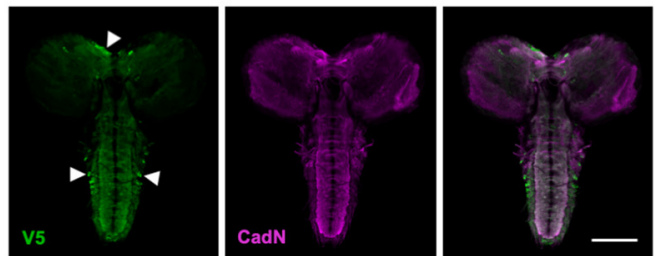

C
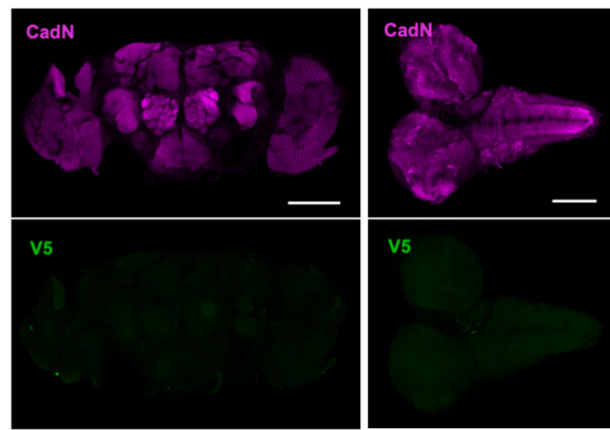

v5
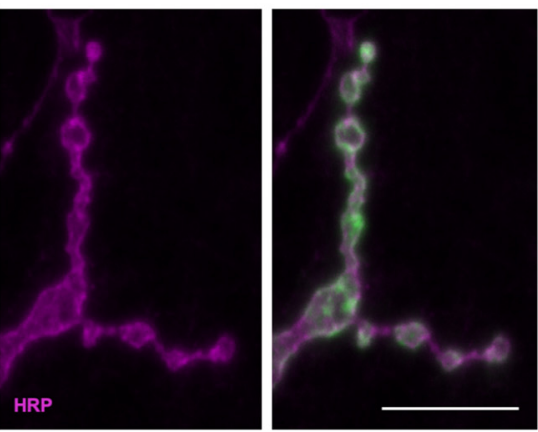

Figure 3. CG31030 is addressed to synaptic areas. $\boldsymbol{A}, \boldsymbol{B}$, Anti-V5 immunostaining in the CG31030 ${ }^{\mathrm{V} 5}$ strain revealed that CG31030 is mainly addressed to synaptic areas in the adult brain $(\boldsymbol{A})$ and larval CNS $(\boldsymbol{B})$, as indicated by its colocalization with the presynaptic marker CadN. Green fluorescence outside synaptic areas reveal that cell bodies are also faintly marked by the V5 antibody, with a few of them showing a bright signal (arrowheads). OL, optic lobe; AL, antennal lobe; SO, subesophageal ganglion. $\boldsymbol{C}$, CadN and V5 immunostaining of adult brain (left panels) and larval CNS (right panels) from $w^{1118}$ control flies. No signal was detected with the anti-V5 antibody in the absence of V5-tagged protein. $\boldsymbol{D}$. Anti-V5 antibody also labels the neuromuscular junction of CG31030 ${ }^{\mathrm{V} 5}$ larvae, where it co-localizes with anti-HRP immunostaining that specifically marks neuronal membranes, confirming CG31030 synaptic localization. Scale bars: $100 \mu \mathrm{m}(\boldsymbol{A}-\boldsymbol{C})$ and $10 \mu \mathrm{m}(\boldsymbol{D})$.

Vha100-1 and VhaAC39-1 code for subunits a and $d$ of the $\mathrm{V}_{0}$ domain, respectively (Vasanthakumar and Rubinstein, 2020). The subunit $a$ is encoded by five different genes in Drosophila and constitutes the proton port of the pump (Collins and Forgac, 2020). Among these five isoforms, Vha100-1 has been shown to be specifically required in neurons and present at the synapse (Hiesinger et al., 2005). Coimmunostaining experiments confirmed that this protein colocalizes with CG31030 at the larval neuromuscular junction (Fig. $4 C, D$ ). Subunit $d$ of $\mathrm{V}_{0}$ is encoded by two Drosophila genes: VhaAC39-1 and VhaAC39-2, and only the first co-immunoprecipitated with CG31030. According to FlyAtlas, VhaAC39-1 is expressed in many tissues and enriched in the brain, while VhaAC39-2 seems to be mostly found in testis and salivary glands. For both $V_{0}$ subunits, CG31030 thus coprecipitated with the likely neuronal isoform. The co-immunoprecipitated V-ATPase subunit which appeared to be the most enriched in the CG31030 55 sample was, interestingly, the accessory subunit ATP6AP2, suggesting a possible direct interaction between this protein and CG31030 (Table 3). These experiments therefore reinforce the hypothesis that CG31030 directly interacts with the neuronal V-ATPase complex, and more specifically with $\mathrm{V}_{0}$ since all detected partners belong, or interact, with this domain.

Similarly, the mouse protein ATP6AP1L/AC45RP, a vertebrate homolog of CG31030, has recently been shown to interact selectively with $\mathrm{V}_{0}$ domain subunits of the neuronal V-ATPase (Jansen et al., 2021). In addition, these authors showed that strong in vitro upregulation $(\sim 7$-fold $)$ of this gene resulted in an increased transcript level of some $V_{0}$ subunit, while a moderate one ( $\sim 2.6$-fold) had no effect except on ATP6AP2. We thus wondered whether CG31030 upregulation or downregulation could affect $V_{0}$ subunit expression in vivo. Using a pan-neuronal driver, we were able to increase CG31030 transcript levels, but only up to $\sim 3.5$ fold in an in vivo wild-type context, suggesting that transcript abundance is regulated posttranscriptionally. This moderate upregulation did not induce any significant 
A

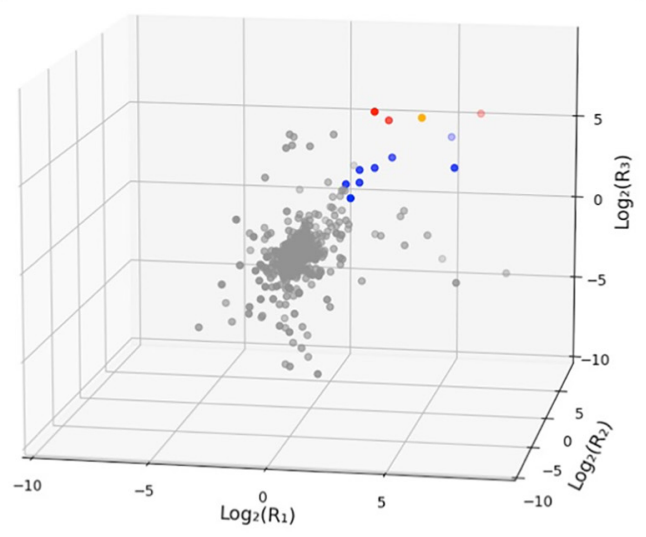

CG31030

- Proteins with $\log _{2}(R)>1$ in all 3 experiments

V-ATPase subunits with $\log _{2}(R)>1$

Proteins with $\log _{2}(R)<1$

B

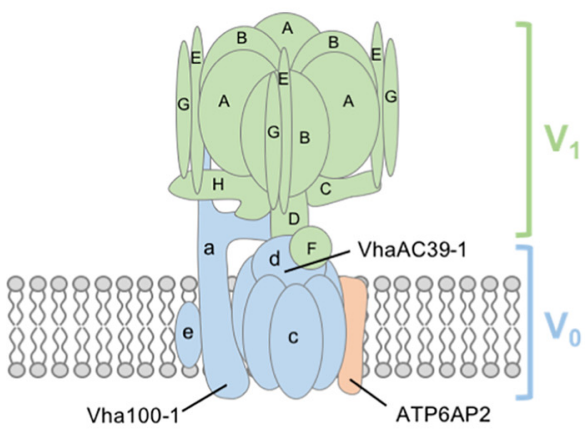

C
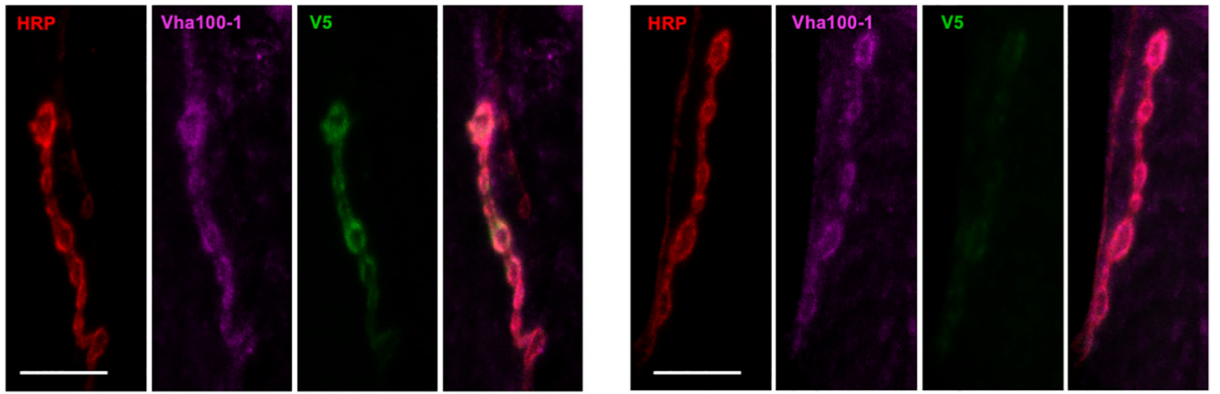

Figure 4. CG31030 co-immunoprecipitates with V-ATPase subunits. $\boldsymbol{A}$, Scatter plot of proteins identified by nano LC-MS/MS in three independent co-immunoprecipitation experiments using anti-V5 antibodies. $R_{1}, R_{2}$ and $R_{3}$ represent the abundance ratio of proteins identified in adult head extracts from $C G 31030^{\sqrt{5}}$ over $w^{1118}$ control in experiments 1,2 and 3 , respectively. $\log _{2}(R)=1$ corresponds to a 2 -fold abundance difference. A total of 12 proteins were found to be at least twice as abundant in CG31030 ${ }^{\mathrm{v} 5}$ as in the control in all three experiments (red or blue dots on the graph). One of them is the immunoprecipitation target CG31030 (yellow dot) and three of these proteins belong to the V-ATPase complex (red dots). A list of these 12 proteins with their $\log _{2}(\mathrm{R})$ values is provided in Table 3. B. Standard model of the Drosophila V-ATPase complex showing structure of the V1 and V0 domains and the predicted localization of the three subunits that co-immunoprecipitated with CG31030. C, Co-localization of CG31030-V5 with Vha100-1 at the larval neuromuscular junction. Larval muscles were labeled with an anti-HRP antibody to mark neuronal membranes. CG31030 has a similar localization to that of Vha100-1, the synaptic isoform of subunit a of the $V_{0}$ domain. $D$, In $w^{1118}$ control flies, no specific signal could be detected at the neuromuscular junction with the anti-V5 antibody. Scale bars: $10 \mu \mathrm{m}(\boldsymbol{C}, \boldsymbol{D})$.

changes in transcript levels of $\mathrm{V}_{0}$ subunits, but possibly it was not sufficient to see such effects (Fig. 5A). Conversely, we performed RT-qPCR experiments on extracts from Drosophila heads expressing CG31030 RNAi3 in all neurons compared with controls, and here again, we could not find any significant change in $\mathrm{V}_{0}$ subunit expression level in knock-down context (Fig. 5B). Notably, VhaAC45 is expressed at a similar level in CG31030 knock-down flies and in controls. The homology between CG31030 and VhaAC45 could raise the question of a potential functional redundancy of the two proteins. However, the lethal phenotype and the highly reduced longevity occurring when CG31030 RNAi.1/2 or CG31030 RNAi.3 are expressed in all neurons, respectively, suggest that
VhaAC45, though still present, is unable to substitute for CG31030.

\section{CG31030 knock-down impairs synaptic vesicle acidification}

Because CG31030 appeared to be mainly localized in synaptic areas, we chose to look at the physiological effect of its disruption at the Drosophila larval neuromuscular junction, a model that has contributed to elucidate many essential synaptic processes. At synaptic nerve endings, a prominent role of the V-ATPase is to acidify the lumen of synaptic vesicles, the electrochemical gradient generated providing the driving force to load 
Table 3: List of the genes encoding proteins that co-immunoprecipitated with CG31030 in Drosophila head extracts

\begin{tabular}{lllll}
\hline Gene symbol $^{1}$ & FlyBase gene ID & $\log _{2}\left(R_{1}\right)$ & $\log _{2}(R 2)$ & Log $_{2}\left(R_{3}\right)$ \\
\hline ATP6AP2 & FBgn0037671 & 6.154281057 & 9.481786693 & 5.17436482 \\
Vha100-1 & FBgn0028671 & 1.499248537 & 8.012982519 & 5.43599503 \\
VhaAC39-1 & FBgn0028665 & 2.104797507 & 8.293264658 & 4.86741949 \\
CG31030 & FBgn0051030 & 3.669278876 & 7.960835791 & 5,19465574 \\
Twdl $\beta$ & FBgn0033658 & 1.642952792 & 3.068640973 & 2.35207192 \\
Ccp84Ag & FBgn0004777 & 1.543972087 & 1.326485589 & 3.86392045 \\
CG13627 & FBgn0039217 & 5.738384187 & 4.008318188 & 3.18433108 \\
mfas & FBgn0260745 & 1.596554441 & 3.368384477 & 3.03376685 \\
CG16820 & FBgn0032495 & 4.77051909 & 9.784086057 & 2.60280541 \\
Cpr64Ab & FBgn0035511 & 1.08994458 & 2.758609792 & 3.10103817 \\
CG14752 & FBgn0033307 & 2.21698759 & 3.69458382 & 3.39808341 \\
CG15615 & FBgn0034159 & 2.77101281 & 5.12362053 &
\end{tabular}

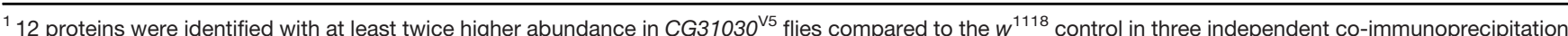
experiments with anti-V5 antibodies, followed by mass spectrometry analysis. Among these proteins, three are known to be constitutive or accessory subunits of the V-ATPase complex: ATP6AP2, Vha100-1 and VhaAC39-1.

and concentrate the neurotransmitters. Thus, a malfunction of synaptic V-ATPase should induce a decrease of neurotransmitter concentration inside the vesicles, potentially resulting in an altered synaptic transmission. To test this hypothesis, we co-expressed each of the two strongest CG31030 RNAi constructs together with VMAT-
pHluorin, a pH-sensitive probe targeted to synaptic vesicles (Wu et al., 2013), in larval motoneurons using the glutamatergic driver OK371-Gal4. Both RNAi1 and RNAi2 induced a lethal phenotype at pupal stage in these conditions. VMAT-pHluorin is an ecliptic pHluorin that is fluorescent at neutral $\mathrm{pH}$, and gets quenched, by protonation, at

A

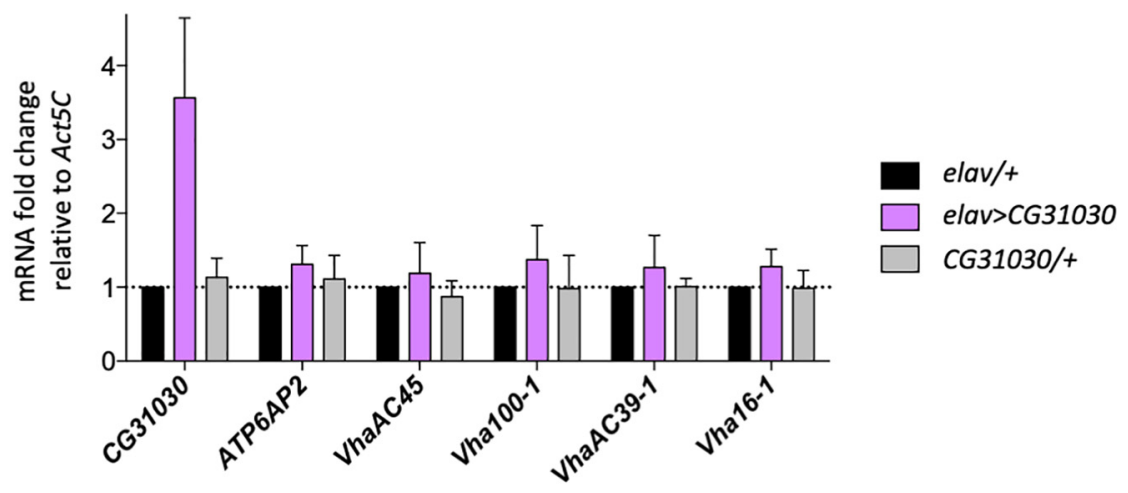

B

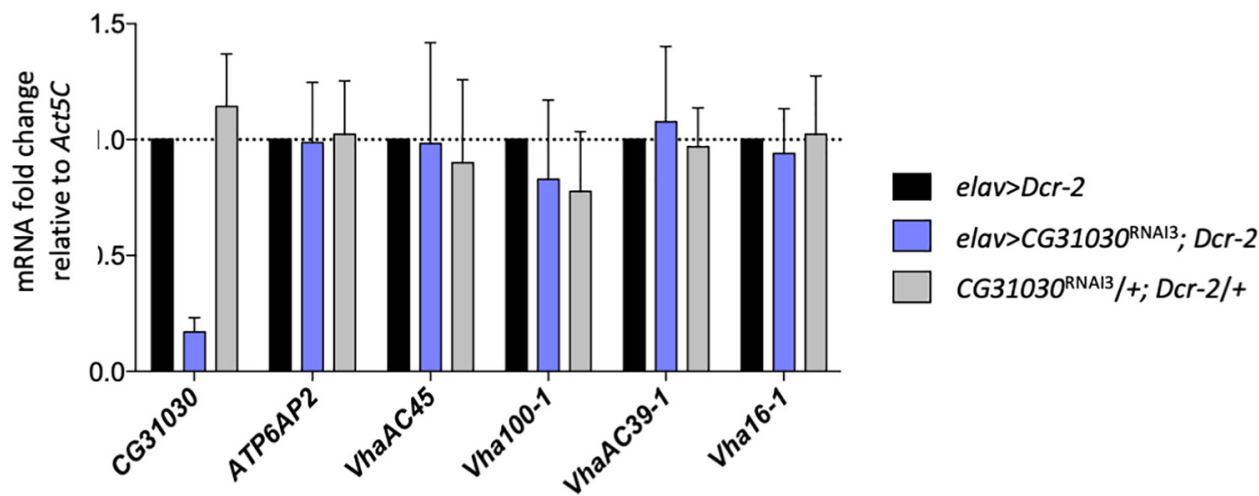

Figure 5. Change in CG31030 expression does not alter V-ATPase subunit transcript levels. $\boldsymbol{A}, \boldsymbol{B}$, CG31030 upregulation (A) or knock-down $(\boldsymbol{B})$ in vivo did not significantly affect adult head transcript levels of several V-ATPase $V_{0}$ subunits. Results of three independent RT-qPCR experiments. Means and SD are displayed on the graph. 
acidic pH (Miesenböck et al., 1998). Thus, in control condition, VMAT-pHluorin should not be fluorescent in synaptic vesicles, whose $\mathrm{pH}$ is at $\sim 5.5$, but only when externalized on the presynaptic membrane during exocytosis, and so, in contact with the more neutral synaptic cleft milieu. In the case of an acidification defect of synaptic vesicles, the probe could be fluorescent both in synaptic vesicles, where $\mathrm{pH}$ would be abnormally high, and on the presynaptic membrane (Fig. 6A, central panel).

In order to evaluate the ratio of the internal fluorescence (from synaptic vesicles) over the external fluorescence (from the presynaptic membrane) at the neuromuscular junction of CG31030 knock-down larvae compared with controls, we first quenched the external signal by replacing the physiological milieu by an identical one with $\mathrm{pH}$ adjusted to 5.5 (Fig. 6A, left panel). This operation resulted in only the internal signal being conserved. In controls, this meant that all signal was abolished, as expected because the synaptic vesicles were normally acidified (Fig. 6B, left panel). In contrast and strikingly, a residual signal was still visible in this acidic milieu in both RNAi1 and RNAi2 knock-down larvae (Fig. 6B, right panel). Quantification of the ratio of fluorescence area in acidic milieu over neutral milieu showed that $\sim 37 \%$ of the total signal remained visible in the RNAi larvae after external quenching (Fig. $6 C, D$ ).

To verify that the residual signal seen in knock-down larvae was indeed coming from inside vesicles, the opposite strategy was used: instead of quenching the outside signal, we revealed all the internal one by collapsing the $\mathrm{pH}$ gradient of synaptic vesicles (Fig. $6 A$, right panel). To do so, we replaced the physiological milieu by an ammonium solution, as previously described (Poskanzer and Davis, 2004). This solution had the same composition except that $50 \mathrm{~mm} \mathrm{NaCl}$ were replaced by $50 \mathrm{~mm} \mathrm{NH}_{4} \mathrm{Cl}$. Ammonium and ammonia being in equilibrium $\left(\mathrm{NH}_{4}{ }^{+} \rightleftarrows\right.$ $\mathrm{NH}_{3}+\mathrm{H}^{+}$), uncharged ammonia crosses membranes and binds to protons leading to an alkalization of vesicle lumen $\mathrm{pH}$. This increase of vesicular $\mathrm{pH}$ is maintained during $\mathrm{NH}_{4} \mathrm{Cl}$ exposure. In control condition, this gradient collapse should reveal the VMAT-pHluorin probe present in synaptic vesicles and thus highly increase the fluorescent signal. On the contrary, in the case of an acidification defect, the signal should remain fairly stable since synaptic vesicles are already fluorescent. Results were consistent with this hypothesis: while the fluorescence of controls increased $\sim 3$-fold in the $\mathrm{pH}$ collapsing ammonium solution, the signal in CG31030 knock-down larvae hardly rose by $10-20 \%$ depending on the RNAi construct (Fig. 6E-G). Taken together, these results indicate that CG31030 knock-down significantly decreases protons concentration in synaptic vesicles of motoneuron terminals.

\section{CG31030 downregulation decreases larval locomotor performance}

A reduced $\mathrm{pH}$ gradient of synaptic vesicles in larval motoneurons could alter synaptic transmission and, consequently, the larval locomotor behavior. To assess locomotion, we recorded the spontaneous crawling of third-instar larvae expressing CG31030 RNAi1 or RNAi2 in motoneurons with OK371-Gal4 on an agar plate with no food source for $2 \mathrm{~min}$ periods. We first checked that the average length and width of knock-down larvae were not significantly different from those of control larvae (Fig. 7A). Tracking was then performed using the FIMtrack software (Risse et al., 2014), allowing measurement of the total distance traveled and of the stride size, defined as the distance crawled during one peristaltic wave of muscle contraction, and duration (Fig. 7B). The knock-down larvae obviously moved less than controls on the plate, as confirmed by their trajectory maps (Fig. 7C), traveling a distance only half as long as controls with both RNAi (Fig. 7D).

In contrast, the stride size actually showed little or no difference, depending on the RNAi, between knock-down larvae and controls (Fig. 7E). Instead, we found that the stride duration, which is the time necessary to accomplish one peristaltic wave, was significantly longer in knockdown animals compared with controls for both RNAi (Fig. $7 F$ ). These results suggest that the knock-down larvae may need about twice as much time as controls to reach the required level of muscular contraction to accomplish one stride. This could be a compensatory mechanism to adapt to a lower amount of neurotransmitter released in response to motor nerve stimulation, as would be expected if the synaptic vesicles were less filled in CG31030 knock-down context.

\section{Quantal size is reduced in CG31030 knock-down larvae}

The locomotor deficit of CG31030 knock-down larvae could originate from an impairment of synaptic transmission at the neuromuscular junction or a defect in the central control of motor behavior, or both. Fluorescent FM-dye loading/unloading experiments can be used to monitor potential defects in endocytosis or exocytosis of synaptic vesicles at the larval neuromuscular junction (Verstreken et al., 2008). We therefore assessed whether the synaptic vesicle cycle induced by $\mathrm{KCl}$ stimulation was affected by CG31030 downregulation, as this could well have an impact on neurotransmission and locomotion. Representative images of neuromuscular junctions from control and knockdown larvae after FM-dye loading and unloading are shown in Figure $8 A, B$, respectively. The larvae expressing CG31030 RNAi1 or RNAi2 in motoneurons, were apparently able to load and unload the dye in synaptic junctions similarly as controls (Fig. $8 C, D$ ). The percentage of previously loaded FM4-64 dye released during the unloading phase was also comparable for control and knock-down larvae (Fig. 8E,F). This result indicates that the synaptic vesicle cycle, and in particular the exocytosis machinery, were apparently not impacted by CG31030 inactivation.

To determine whether neurotransmission was otherwise affected by CG31030 knock-down, we conducted electrophysiological recordings to measure the quantal size, which is the postsynaptic response to the release of one synaptic vesicle. We expressed CG31030 RNAi1 or RNAi2 in motoneurons of third-instar larvae with the OK371-Gal4 driver, and recorded spontaneous mEPSPs intracellularly from ventral longitudinal abdominal muscle 
A
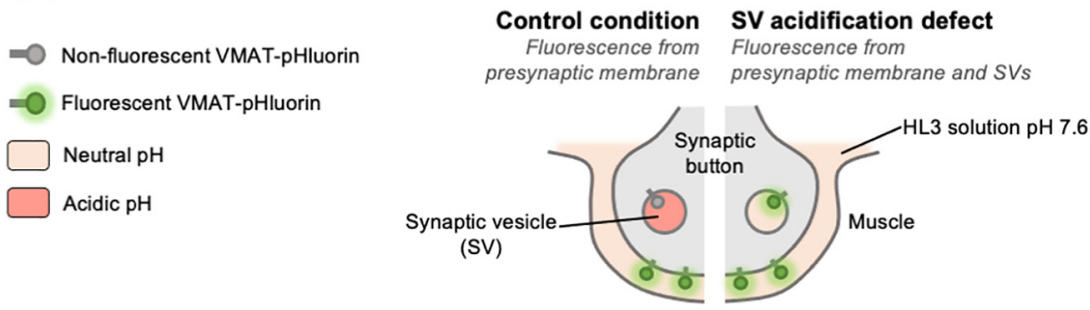

HL3 solution $\mathrm{pH} 5.5$

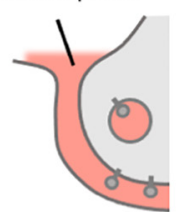

Control condition No fluorescence

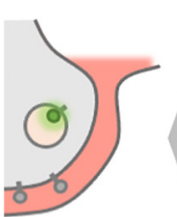

SV acidification defect Fluorescence from SVS

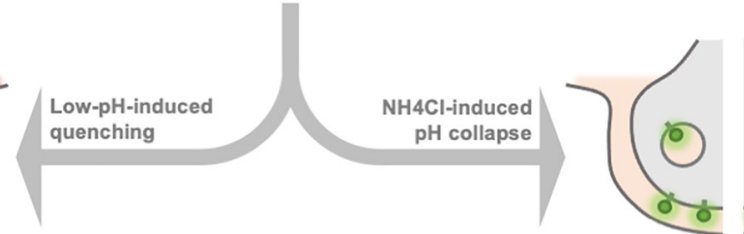

Control condition Fluorescence from presynaptic membrane
$\mathrm{HL} 3-\mathrm{NH}_{4} \mathrm{Cl} \mathrm{pH7.6}$

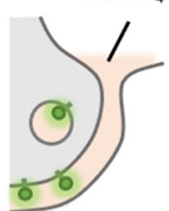

SV acidification defect Fluorescence from presynaptic membrane and SV
B

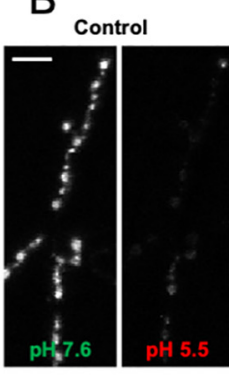

E

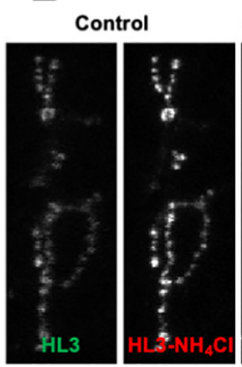
CG31030 knockdown

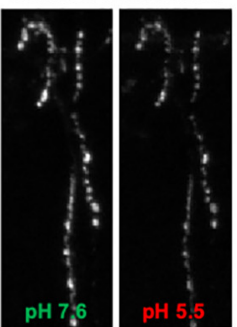

C

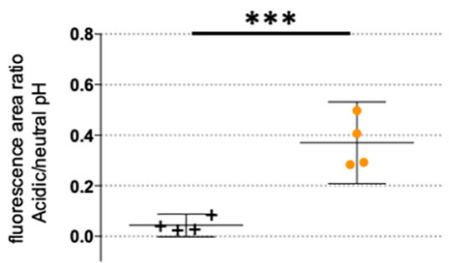

$\mathrm{F}$ CG31030 knockdown

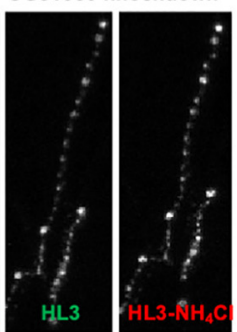

D

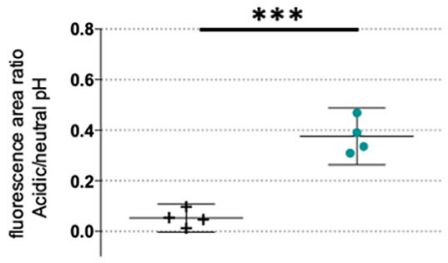

G

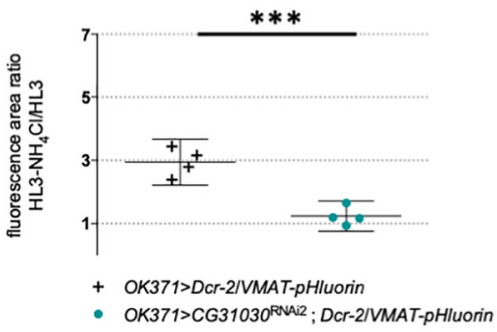

Figure 6. CG31030 knock-down larvae have a synaptic vesicle acidification defect. $\boldsymbol{A}$, Schematic representation of the protocols used to assess relative acidity levels of synaptic vesicles at the larval neuromuscular junction. Top center diagram, In control conditions, fluorescence can be emitted by VMAT-pHluorin in the presynaptic membrane but not in synaptic vesicles since their lumen is acidified. In case of defective synaptic vesicle acidification, both the presynaptic membrane and synaptic vesicles should emit fluorescence. Left diagram, The fluorescence emitted by VMAT-pHluorin in the presynaptic membrane can be quenched by replacing the extracellular medium with an acidic HL3 solution. This quenching should result in an almost complete extinction of the signal in control flies, in which synaptic vesicles are normally acidified, while a residual signal is expected to be visible in flies having a synaptic vesicle acidification defect. Right diagram, Replacement of $50 \mathrm{~mm} \mathrm{NaCl}$ by $50 \mathrm{~mm} \mathrm{NH}_{4} \mathrm{Cl}$ in the neutral HL3 solution should lead to a collapse of the $\mathrm{pH}$ gradients because of the free diffusion of $\mathrm{NH} 3$ in membranes, so that fluorescence will be emitted both by the presynaptic membrane and synaptic vesicles both in control and mutant conditions. $\boldsymbol{B}$, Representative pictures showing the effect of perfusing an acidic HL3 solution on VMAT-pHluorin fluorescence in control and CG31030 knock-down larvae. C, $\boldsymbol{D}$, Quantification of the ratio of the fluorescence level at $\mathrm{pH} 5.5$ over the original signal at $\mathrm{pH}$ 7.6. Whereas the low $\mathrm{pH}$ extinguished fluorescence in control flies, $\sim 37 \%$ of the signal persisted after quenching in CG31030 knock-down larvae using two different RNAi constructs. $\boldsymbol{E}$, Representative pictures showing the effect of collapsing the synaptic vesicle pH by perfusing $\mathrm{HL}^{-} \mathrm{NH}_{4} \mathrm{Cl}$ in control and CG31030 knock-down larvae. $\boldsymbol{F}, \boldsymbol{G}$, Quantification of the ratio of the signal in $\mathrm{HL} 3-\mathrm{NH}_{4} \mathrm{Cl}$ over the original signal in HL3 showed that fluorescence increased $\sim 3$-fold in controls while it only rose by 10-20\% depending on the RNAi in CG31030 knock-down larvae. Results of four independent experiments, with three to five larvae analyzed per genotype in each experiment. Unpaired Student's $t$ test; ${ }^{\star} p<0.05,{ }^{* \star} p<0.001$. Mean values with $95 \%$ confidence intervals are reported on the graphs. Scale bar: $15 \mu \mathrm{m}(\boldsymbol{B})$. 
A
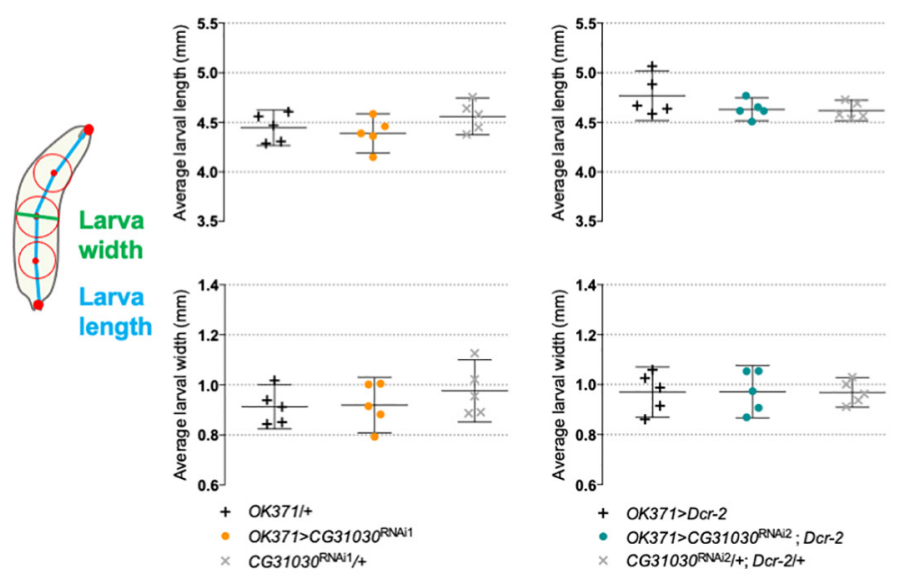

C
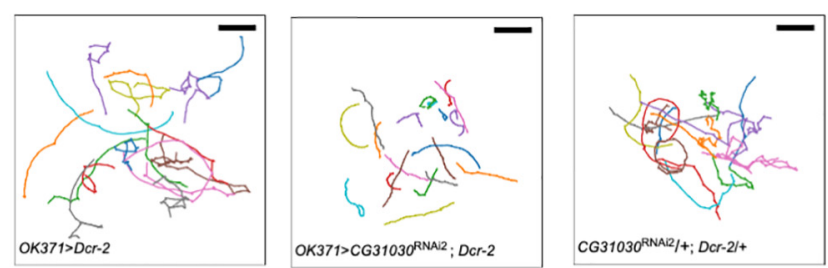

B
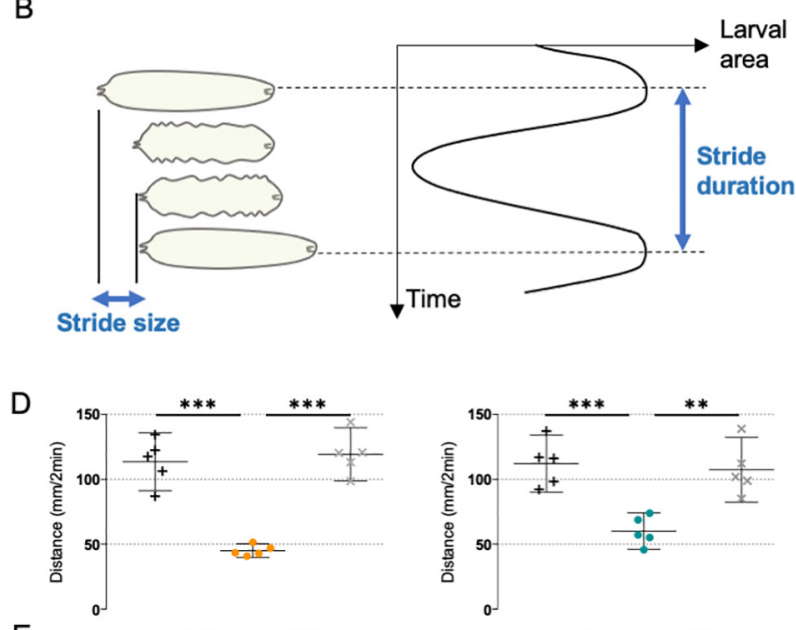

E

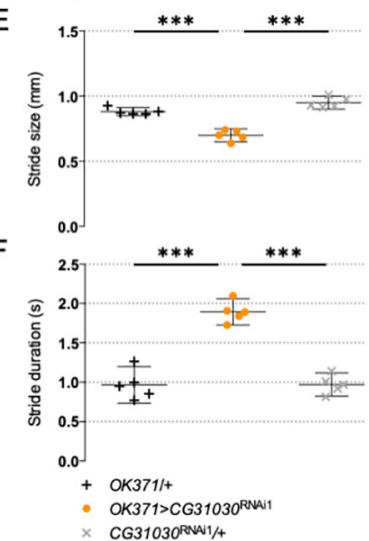

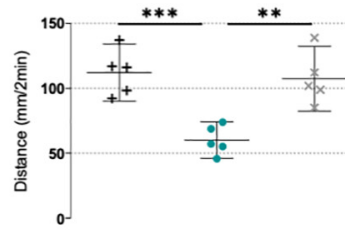

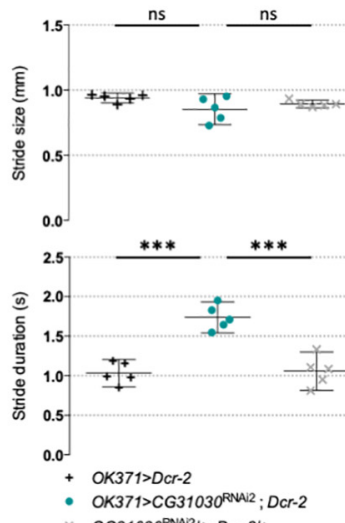

Figure 7. CG31030 downregulation in motoneurons decreases larval locomotor performance. $\boldsymbol{A}$, CG31030 knock-down in motoneurons did not significantly alter larval size. Larval length is defined as the spine length from head to tail, while larval width is the diameter of the mid-spine circle. Average larval length and width were not significantly different between animals expressing CG31030 RNAi1 (left panels) and RNAi2 (right panels) in motoneurons and controls. B. Schematic representation of the successive phases of larval locomotion. Stride size is defined as the distance crawled during one peristaltic wave of muscle contraction, while stride duration is the time necessary for the completion of one peristaltic wave. $\boldsymbol{C}$, Locomotor trails of individual larvae recorded over a period of $2 \mathrm{~min}$. Larvae expressing CG31030 RNAi in motoneurons show reduced spontaneous movements (middle panel) compared with the driver (left panel) and effector (right panel) controls, respectively. Scale bar: $25 \mathrm{~mm}$. $\boldsymbol{D}$, Quantification of the traveled distances confirmed that knocking down CG31030 in motoneurons with two different RNAi induced significant locomotor defects. $\boldsymbol{E}, \boldsymbol{F}$, Stride size $(\boldsymbol{E})$ appears to be less affected than stride duration $(\boldsymbol{F})$ in the knocked-down larvae. Result of five independent experiments, with three to four larvae analyzed per genotype in each experiment. One-way ANOVA with Dunnett's post hoc test for multiple comparisons; ${ }^{* *} p<0.01,{ }^{* *} p<0,001$. Mean values with $95 \%$ confidence intervals are reported on the graphs.

6 of segment A3 (Fig. 9A). These muscles are innervated by synaptic boutons that are clearly marked by OK371Gal4, as shown by the co-localization of membrane-associated GFP and the postsynaptic marker Dlg in OK371> $m C D 8:: G F P$ flies (Fig. 9B). Representative amplitude distributions of mEPSPs in a control and RNAi1 larvae are shown in Figure 9C. Quantal analysis of recorded events confirmed that both RNAi1 and RNAi2 knockdown larvae have a significantly reduced quantal size compared with controls (Fig. 9D), suggesting a decrease in glutamate vesicular uptake, and potentially linking vesicle acidification defect and locomotor impairment. The V-ATPase dysfunction in synapses of CG31030 knock-down larvae could apparently lead to incomplete loading of vesicles, thus decreasing the amount of neurotransmitter released per unit of time during a peristaltic wave, and potentially slowing down larval locomotion. Although the mean frequency of mEPSPs of both RNAi1 and RNAi2 larvae appeared lower than controls, this effect was not statistically significant (Fig. 9E). This may suggest that CG31030 knock-down did not significantly increase the number of unacidified vesicles empty of neurotransmitter.

Finally, we also looked at the morphology of the neuromuscular junctions in CG31030 knock-down and control animals by measuring the number and diameter of synaptic boutons innervating the ventral longitudinal abdominal muscle 6 in the segment A3. The overall number and mean size of boutons were not significantly affected between the different genotypes (Fig. 10A-D). However, we observed that knock-down larvae exhibited a reduced number of boutons with a 
A

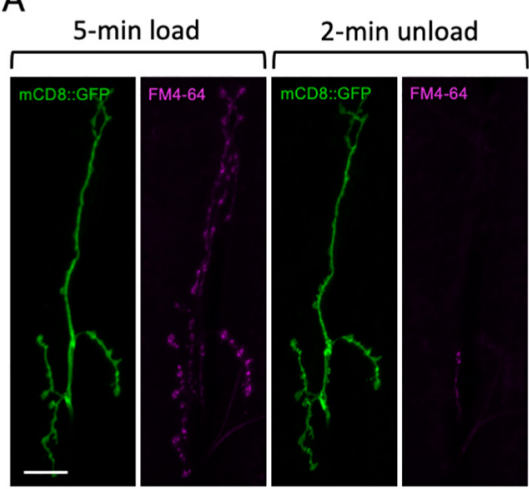

C

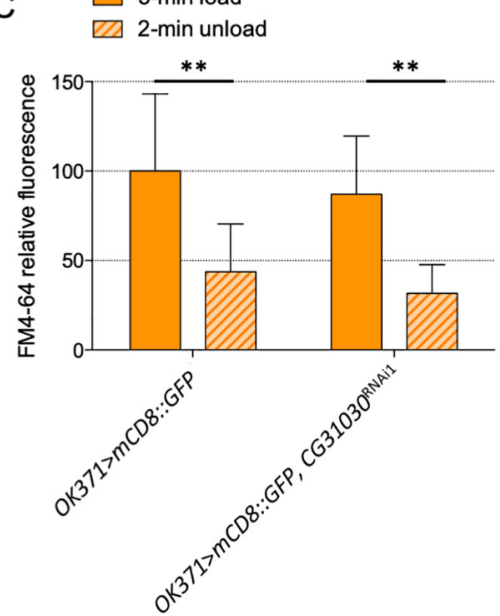

E

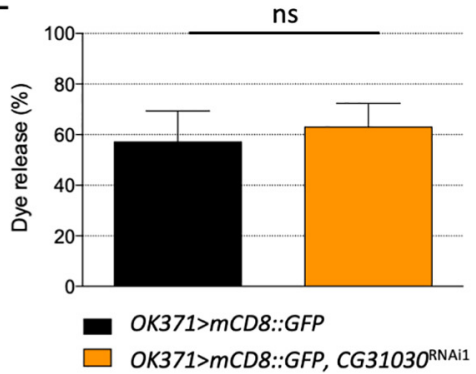

B

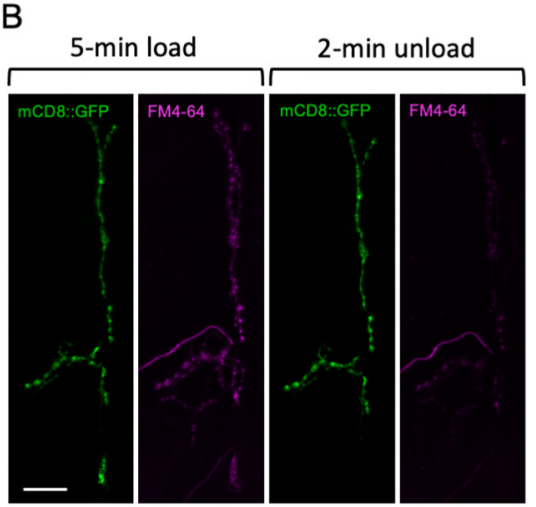

D

D $\square$-min load
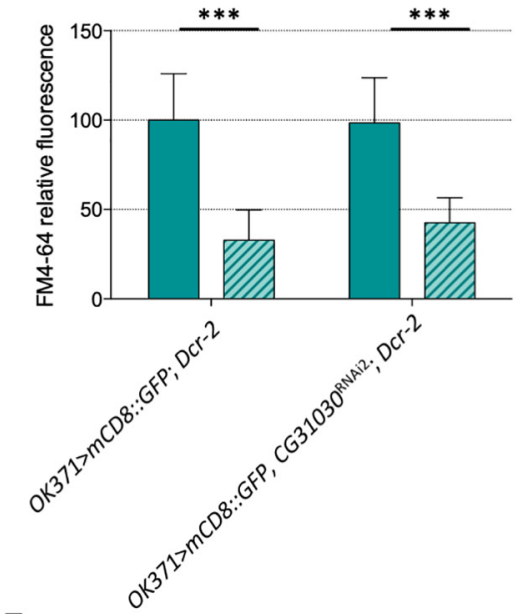

$\mathrm{F}$

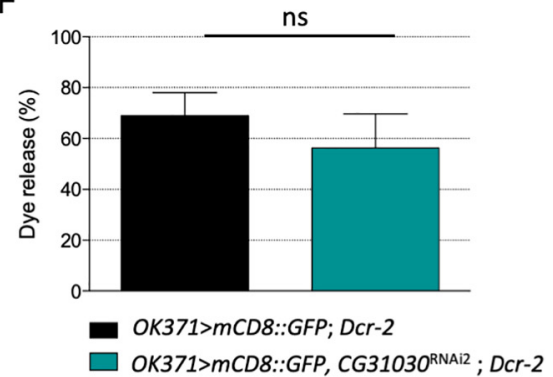

Figure 8. Lack of effect of CG31030 knock-down on the synaptic vesicle cycle at the larval neuromuscular junction. $\boldsymbol{A}, \boldsymbol{B}$, Representative images of mCD8::GFP-labeled neuromuscular junctions of a control $(\boldsymbol{A})$ and a knock-down $(\boldsymbol{B})$ larva after loading and unloading of the fluorescent FM4-64 dye. C, D, Nerve terminals from larvae expressing CG31030 RNAi1 (C) or RNAi2 (D) were able to both load and unload the fluorescent FM4-64 dye as efficiently as controls. No significant difference was observed between controls and knock-down animals during the loading and the unloading phases. Fluorescence was normalized relative to the level in control larva synapses after 5-min loading. $\boldsymbol{E}, \boldsymbol{F}$, The percentage of previously loaded FM4-64 dye released during the unloading phase was similar for control and knock-down larvae when using either RNAi1 $(\boldsymbol{E})$ or RNAi2 $(\boldsymbol{F}) ;{ }^{* *} p<0.01,{ }^{* \star *} p 0.001$, ns: not significant.

diameter superior to $4 \mu \mathrm{m}$ for both RNAi1 and RNAi2 (Fig. 10E,F). The fact that these large boutons only represented a small fraction of the total explains why the average number or mean diameter of boutons were not altered in knock-down larvae. It is therefore unlikely that this size variation could result, by itself, in the significant decrease in quantal size and strong locomotor impairment induced by CG31030 downregulation. Neuronal activity is known to influence synaptic growth at the neuromuscular junction (Menon et al., 2013), so the lower number of larger boutons in knock-down animals could be a consequence of the impaired synaptic transmission resulting from the quantal size decrease. Alternatively, the lower number of high diameter boutons could also be a modest contributor to the observed quantal size decrease, as larger boutons have been shown to harbor bigger synaptic vesicles associated with higher quantal content (Karunanithi et al., 2002). 
A

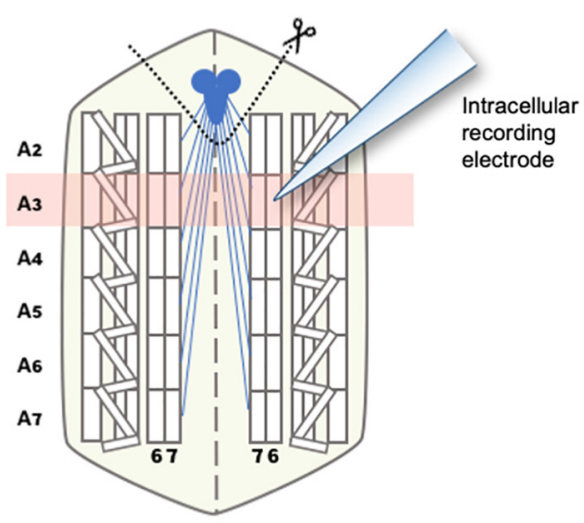

c
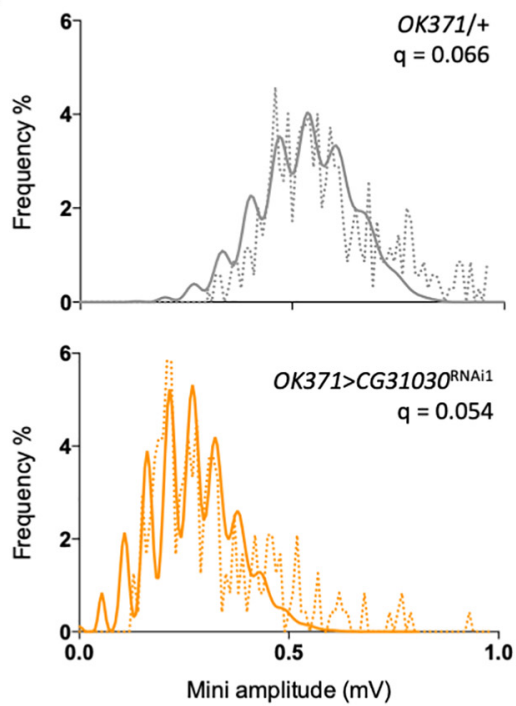

$E$

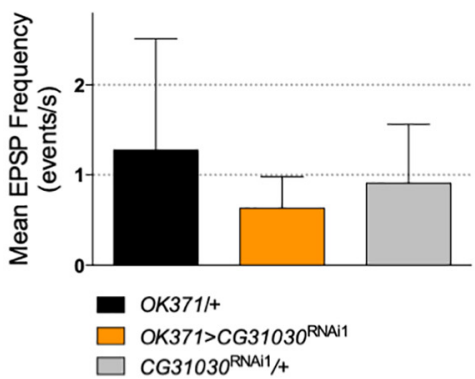

B
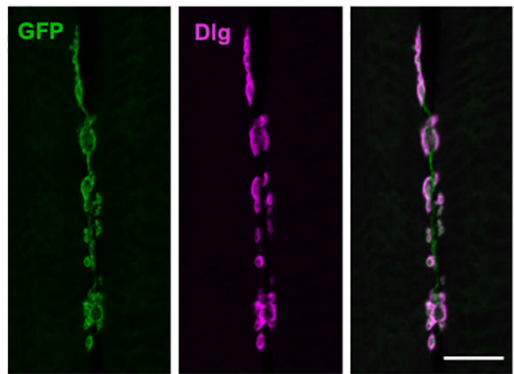

D
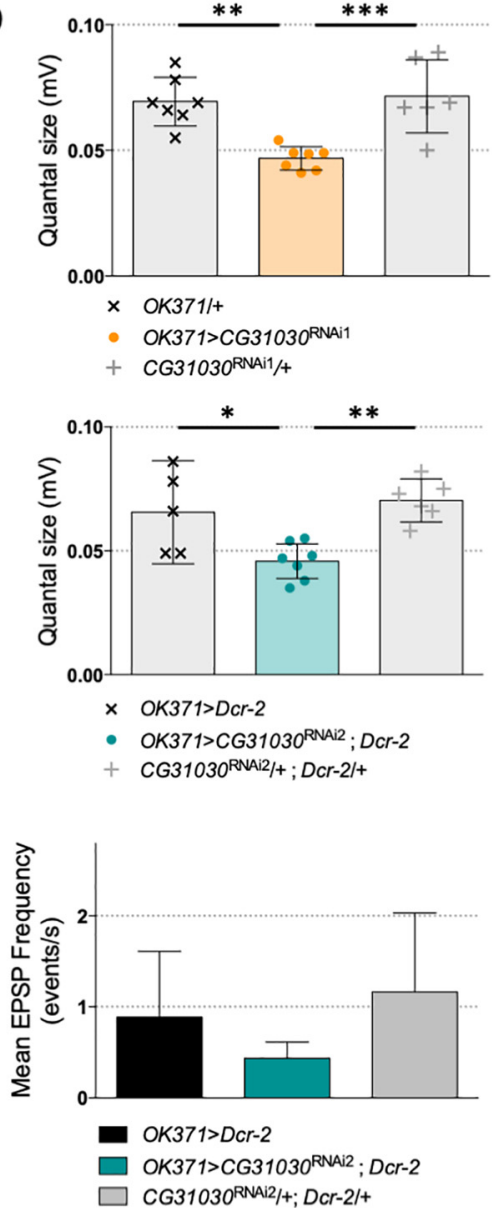

Figure 9. Synaptic quanta size is reduced in CG31030 knock-down larvae. $\boldsymbol{A}$, Schematic representation of a dissected larval fillet. Spontaneous miniature EPSPs (mEPSPs) were recorded intracellularly from the ventral longitudinal abdominal muscle 6 in segment A3. B, Expression of membrane-associated mCD8::GFP with the glutamatergic driver OK371-Gal4 strongly labels the presynaptic nerve endings at the neuromuscular junction of muscles 6-7 in segment A3. The scaffolding protein Dlg was used as a postsynaptic marker. Scale bar: $20 \mu \mathrm{m}$. C, Representative distributions of spontaneous mEPSPs recorded in a control larva (top panel, in gray) and a CG31030 RNAi knock-down larva (bottom panel, in orange). The dotted lines represent actually recorded amplitudes and the plain lines the computed theoretical distributions. Genotypes and quantal size (q) are indicated on each graph. $\boldsymbol{D}$, Quantal analysis of recorded events showed that knock-down larvae have a significantly reduced synaptic quanta size compared with controls, both with CG31030 RNAi1 (top panel, in orange) or RNAi2 (bottom panel, in blue). $\boldsymbol{E}$, Although both RNAi1 (left panel) and RNAi2 (right panel) larvae had a lower mean EPSP frequency than controls, this difference was not statistically significant. Six to seven cells recorded per genotype. One-way ANOVA with Dunnett's post hoc test for multiple comparisons; ${ }^{*} p<0.05,{ }^{* *} p<0.01,{ }^{* * *} p<0.001$. Mean values with $95 \%$ confidence intervals are reported on the graphs. 
A

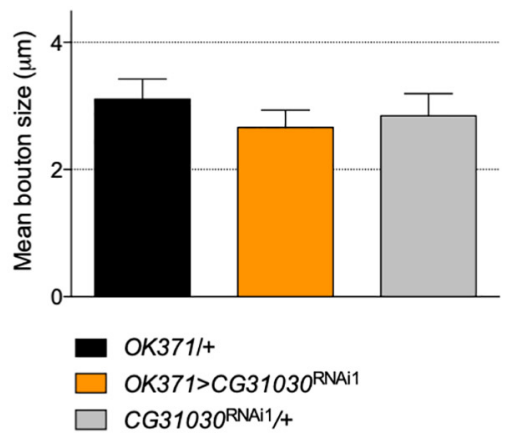

C

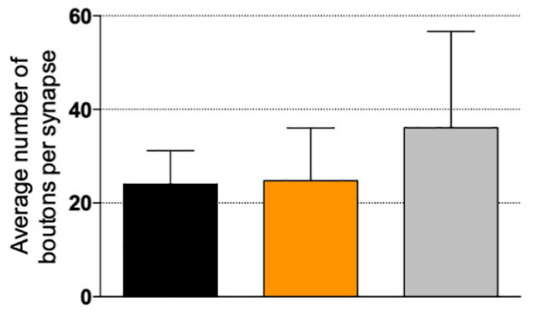

E

$\square \%$ of boutons of diameter $>4 \mu \mathrm{m}$

ए \% of boutons of diameter $<4 \mu \mathrm{m}$

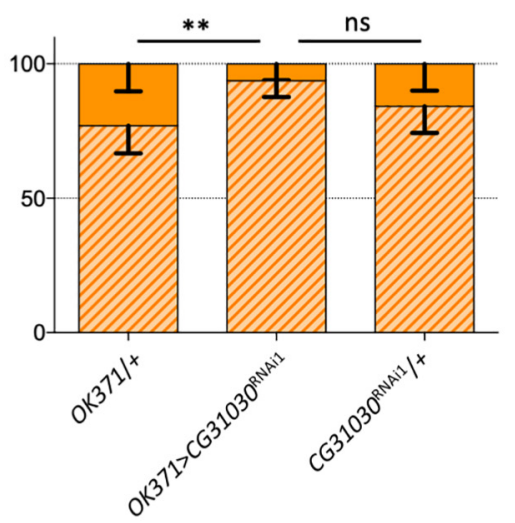

B

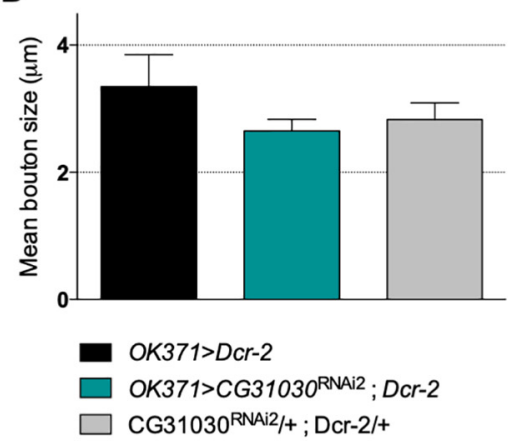

D

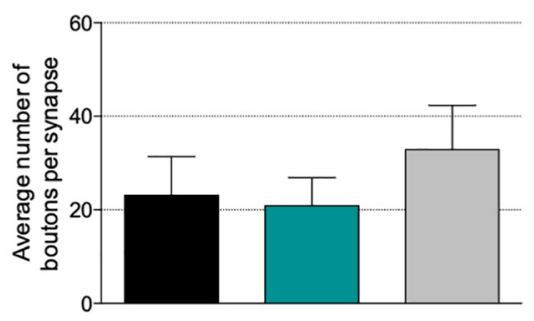

F

$\%$ of boutons of diameter $>4 \mu \mathrm{m}$ W2 \% of boutons of diameter $<4 \mu \mathrm{m}$

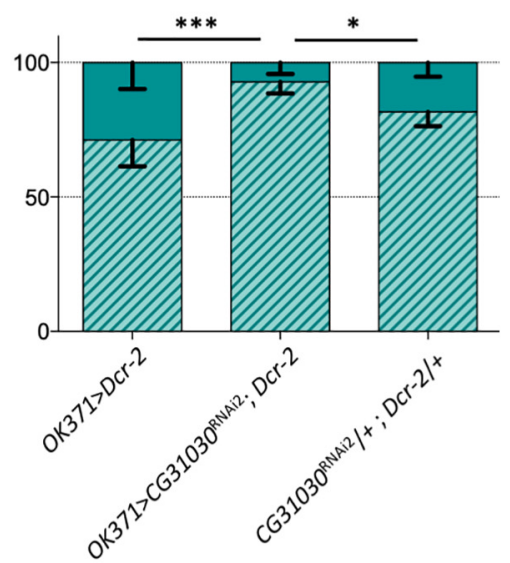

Figure 10. Effect of CG31030 knock-down on synapse morphology at the larval neuromuscular junction. $\boldsymbol{A}-\boldsymbol{D}$, No significant difference was observed in the mean synaptic bouton diameter $(\boldsymbol{A}, \boldsymbol{B})$ or the average number of boutons per synapse $(\boldsymbol{C}, \boldsymbol{D})$ between larvae expressing CG31030 RNAi1 $(\boldsymbol{A}, \boldsymbol{C})$ or RNAi2 $(\boldsymbol{B}, \boldsymbol{D})$ in motoneurons and the controls. $\boldsymbol{E}, \boldsymbol{F}$, Knock-down larvae showed a smaller percentage of boutons with a diameter larger than $4 \mu \mathrm{m}$ for both RNAi1 [E; but not significant (ns) compared with the effector control] and RNAi2 $(\boldsymbol{F}), n=6-8$ synapses per genotype, two-way ANOVA with Dunnett's correction for multiple comparisons; ${ }^{*} p<0.05$, ${ }^{* *} p<0.01,{ }^{* * *} p<0.001$, ns: not significant.

\section{Discussion}

In this study, we investigated the hypothesis that the previously uncharacterized Drosophila protein CG31030 is a specific regulator of the neuronal V-ATPase. At variance with its broadly expressed paralog VhaAC45, we have shown that CG31030 is found mainly, if not only, in neurons. We also provide evidence that CG31030 interacts with two constitutive subunits and one accessory subunit of the V-ATPase, the constitutive ones being also enriched in neurons, and that it is required to have properly acidified synaptic vesicles. This implies that CG31030 is an essential protein for nervous system functioning in Drosophila.

\section{CG31030 is an essential synaptic protein}

In yeast, all V-ATPase subunits are coded by a single gene, with the exception of the $V_{0}$ subunit $a$. The knockout of any of the single-gene subunits all present a similar phenotype: the inability to survive in a neutral pH environment (Nelson, 2003). For subunit $a$, the same phenotype was only achieved in a double-mutant of both isoforms (Manolson et al., 1994). In multicellular organisms, 
mutations of V-ATPase subunits, or accessory proteins, also often lead to a lethal phenotype at various developmental stages, whether in mice (Inoue et al., 1999; Sun-Wada et al., 2000; Schoonderwoert and Martens, 2002b), Caenorhabditis elegans (Lee et al., 2010), or flies (Davies et al., 1996; Allan et al., 2005). Similarly, we found a CG31030 null mutant to be embryonic lethal, and, interestingly, this lethality could be rescued to the adult stage by re-expressing CG31030 in all neurons, showing that the protein is specifically required in this cell type for fly survival. The percentage of adult survivors was about three times less than what would be expected in case of a full rescue (Table 2) and the missing two-thirds likely died at an early developmental stage as no larval lethality was observed. This could be explained by the fact that CG31030 protein re-expression in rescued knock-out flies first required the expression of Gal4 under regulation of the elav promoter, which starts to express rather late in embryos, followed by activation of the UAS sequence upstream the CG31030 insert. So, it is possible that part of the mutant embryos did not survive the delay inherent to this ectopic expression process.

We observed that CG31030 transcripts follows the repartition of the nervous system, with the highest relative abundance in the head. Moreover, pan-neuronal expression of CG31030 $0^{\mathrm{RNAi}}$, the RNAi construct with the weakest effect on fly survival, was sufficient to decrease by $>80 \%$ its level in the brain of adult escapers. These results indicate that CG31030 expression is mainly neuronal, if not even entirely restricted to neurons. In addition, CG31030 cellular localization shows similarity with a synaptic pattern. Cell bodies were also marked, although generally less strongly. Synaptic protein complexes can be assembled in the cell bodies before being transported in axons, and it is the case for the soluble and membranebound domains of synaptic V-ATPase, as was shown in Torpedo (Morel et al., 1998). It is difficult to decide whether the signal coming from cell bodies corresponds to a functional site for CG31030 or rather to newly produced proteins that have not yet been targeted to synapses. However, the prominent signal in synaptic areas led us to assume that CG31030 plays an important role in the synaptic process.

\section{CG31030 is required for proper synaptic vesicle acidification}

In presynaptic terminals, the most abundant organelles are synaptic vesicles, which require acidification to provide the driving force for neurotransmitter loading. This acidification is ensured by the V-ATPase pump, which generates an electrochemical gradient by importing protons into the vesicle lumen, thus creating both a membrane potential $(\Delta \Psi$, inside positive voltage) and a $\mathrm{pH}$ gradient $(\Delta \mathrm{pH}$, acidic lumen). Our experiments showed that the knock-down of CG31030 at the glutamatergic larval neuromuscular junction increased the internal $\mathrm{pH}$ of synaptic vesicles, and so decreased the $\Delta \mathrm{pH}$ component of the electrochemical gradient generated by the $\mathrm{V}$ ATPase. This result is however by itself insufficient to conclude to a dysfunction of the proton pump, since other players have been shown to influence synaptic vesicle $\mathrm{pH}$ gradient downstream V-ATPase activity. For example, cation $/ \mathrm{H}^{+}$exchangers, found on synaptic vesicles, can decrease $\Delta \mathrm{pH}$ while increasing $\Delta \Psi$ by exchanging cations, like $\mathrm{Na}^{+}$or $\mathrm{K}^{+}$, against luminal $\mathrm{H}^{+}$(Takamori, 2016). This activity can be upregulated by increased intracellular concentration of these cations (Huang and Trussell, 2014). Glutamate being negatively charged, its transport across vesicular membrane is predominantly driven by $\Delta \Psi$ (Maycox et al., 1988; Tabb et al., 1992). As a consequence, the increased membrane potential resulting from upregulation of cation $/ \mathrm{H}+$ exchangers actually facilitates glutamate uptake (Goh et al., 2011; Huang and Trussell, 2014). We measured synaptic quanta size by quantal analysis of mEPSPs of CG31030 knock-down larvae and found that it was decreased, contrary to what would be expected in the case of an upregulation of cation $/ \mathrm{H}^{+}$exchanger activity. This apparent decrease of vesicular glutamate concentration was corroborated by its phenotypical consequence, namely the locomotion defect exhibited by knockdown larvae. This suggests that the observed diminution of $\Delta \mathrm{pH}$ is correlated with a diminution of $\Delta \Psi$, thus pointing toward a V-ATPase malfunction. This hypothesis is further supported, physiologically, by the lack of defect in the endocytosis/exocytosis cycling of synaptic vesicles in knockdown larvae, making it unlikely that such a defect could be at the origin of the locomotor impairment, and, at the molecular level, by the co-immunoprecipitation of CG31030 with three V-ATPase subunits of the $\mathrm{V}_{0}$ domain (Vha100-1, VhaAC39-1, and ATP6AP2), all of them being associated with the neuronal V-ATPase. Altogether, these results strongly suggest that CG31030 is a neuronal protein necessary for V-ATPase function in synaptic nerve endings.

Some V-ATPase subunits isoforms in other species have been shown to target specific subcellular compartments, like the Torpedo $V_{0}$ a1 isoform which is found in synaptic V-ATPase and not in neuronal cell bodies (Morel et al., 2003). Similarly, CG31030 could be specifically involved in the regulation of synaptic V-ATPase, or, alternatively, globally act on all neuronal V-ATPase. Specificity level could even be pushed further, since synaptic vesicles are not the only organelles requiring acidification that are localized in synapses. Thus, a synaptic V-ATPase regulator could be devoted to synaptic vesicles as it could be affecting more generally all synaptic V-ATPase complexes, indifferently of their membrane localization. The answer to such questions could help better understand regulations of synaptic transmission, since synaptic VATPase activity is one of the presynaptic modulators of quantal response (Takamori, 2016; Gowrisankaran and Milosevic, 2020).

\section{CG31030 interacts with ATP6AP2 and may regulate V- ATPase domain dynamics}

While accessory subunits directly interact with VATPase domains, other stimulus can indirectly affect activity of the complex, like glucose concentration or serotonin (Sautin et al., 2005; Voss et al., 2007). Co-precipitation of CG31030 with V-ATPase subunits does not necessarily imply a direct interaction, but suggests a minima an 
indirect association with the complex, possibly in a nontransient manner. The impairment in V-ATPase activity induced by CG31030 downregulation rules out the hypothesis of an inhibitory action of this protein on the proton pump. Nevertheless, the precise requirement of CG31030 for activity of the V-ATPase complex still remains unknown to date. Lethality of the mutant could indicate an essential role of CG31030 in V-ATPase function. However, some VATPase regulators and accessory subunits have been found in other signaling pathways, like ATP6AP2 in the renin-angiotensin system (Nguyen, 2010), so we cannot exclude that lethality could be because of CG31030 playing a part in other vital neuronal functions. Signal in axons could also point to a role in targeting the complex to the synapses. The two domains of the V-ATPase, $V_{0}$ and $V_{1}$, are believed to be assembled in cell bodies before being separately transported to synaptic area, $V_{0}$ by fast axonal anterograde transport, most likely directly on new synaptic vesicles, and $V_{1}$ by slow axonal transport like other cytoplasmic synaptic proteins (Morel et al., 1998). CG31030 could be involved in the transport of one of the two domains, and effects of CG31030 knock-down could then result from a decreased synaptic abundance of the affected V-ATPase domain. However, it is believed that only one copy of the V-ATPase complex is sufficient to properly acidify one synaptic vesicle (Takamori, 2016), a consequence of this being that neurotransmitter loading would be an all-or-none process. Consistent with this, mutation of the Drosophila synaptic $\mathrm{V}_{0}$ subunit Vha100-1 does not seem to impact quantal size but rather mEPSP frequency, potentially reflecting the presence of an increased number of empty synaptic vesicles (Hiesinger et al., 2005). Similarly, a reduction in the frequency of spontaneous quantal events with no change in quantal size was observed when the Drosophila vesicular glutamatergic transporter (VGlut) was downregulated, also suggesting that a single copy of VGlut is sufficient for proper loading of a vesicle (Daniels et al., 2006). The fact that CG31030 knock-down changes the quantal size but not significantly mEPSP frequency seems to point toward a role of this accessory protein in V-ATPase efficiency, rather than in a process affecting the abundance of the complex such as synaptic targeting.

In this respect, it is interesting to note that the VATPase protein that more consistently co-immunoprecipitated with CG31030 in our experiments is the accessory subunit ATP6AP2, the fly homolog of human ATP6AP2/ PRR. Interestingly, the vertebrate homolog of CG31030, ATP6AP1/Ac45, has also been shown to interact with ATP6AP2, and the complex they form has been proposed to enable the assembly and disassembly of the catalytic and membrane domains of the V-ATPase in the mammalian brain (Rujano et al., 2017; Abbas et al., 2020). This suggests that CG31030 could similarly play a role in the regulation of these dynamic processes. Indeed, it was shown that the dissociation of the two V-ATPase domains on synaptic vesicles is a necessary step before exocytosis (Bodzeôta et al., 2017). When new vesicles are formed through endocytosis, the two domains then reassemble to start re-acidifying the vesicular lumen, allowing neurotransmitter uptake. Thus, during the synaptic vesicles recycling cycle, $V_{0}$ and $V_{1}$ dynamically alternate between assembled and disassembled states. CG31030 could then be important to facilitate the assembly of the two domains or maintain the assembled state, which may also influence neurotransmitter loading and release in a more continuous way. Our results suggest indeed that modulating V-ATPase activity in presynaptic terminals can finely affect quantal size. Whether such regulation actually occurs in physiological, or pathologic, conditions remains to be established.

Human ATP6AP2/PRR is known to be a Parkinsonism candidate gene and its mutations in humans, mice or flies can lead to cognitive impairment, neurodegeneration and epilepsy (Korvatska et al., 2013; Dubos et al., 2015; Ichihara and Yatabe, 2019). It is therefore of major interest to identify a potential new interactor of ATP6AP2 that is specific to the nervous system, as it could help better understand the consequences of V-ATPase dysregulation on synaptic transmission in pathologic contexts. Accordingly, it would be very interesting to determine whether human ATP6AP1 and/ or ATP6AP1L share a conserved function with Drosophila CG31030 in the nervous system. Like CG31030, the mouse ATP6AP1LAC45RP was recently reported to be restricted to neurons (Jansen et al., 2021), and they are, to date, the only known V-ATPase genes with such tissue specificity. Both proteins also have the particularity of not having a predicted furin cleavage site. ATP6AP1L/AC45RP was shown to be involved in neuronal growth (Jansen et al., 2021), while we observed that CG31030 downregulation also decreased the number of large synaptic boutons at the neuromuscular junction, hinting at a potential functional homology. Owing to its structural and functional similarity with its closest Drosophila homolog, VhaAC45/ATP6AP1, and vertebrates ATP6AP1L/AC45RP, we propose therefore that CG31030 be named VhaAC45-related protein (VhaAC45RP).

In conclusion, our present work identified a specific regulator of neuronal V-ATPase in Drosophila that is required for synaptic vesicle acidification, nervous system functioning and survival. Further study on this protein should provide a more detailed picture of the complex regulations surrounding neuronal V-ATPase specificity, revealing potential therapeutic targets and a better understanding of fundamental processes such as synaptic transmission.

\section{References}

Abbas YM, Wu D, Bueler SA, Robinson CV, Rubinstein JL (2020) Structure of V-ATPase from the mammalian brain. Science 367:1240-1246.

Allan AK, Du J, Davies SA, Dow JA (2005) Genome-wide survey of VATPase genes in Drosophila reveals a conserved renal phenotype for lethal alleles. Physiol Genomics 22:128-138.

Bodzeôta A, Kahms M, Klingauf J (2017) The presynaptic v-ATPase reversibly disassembles and thereby modulates exocytosis but is not part of the fusion machinery. Cell Rep 20:1348-1359.

Brand AH, Perrimon N (1993) Targeted gene expression as a means of altering cell fates and generating dominant phenotypes. Development 118:401-415.

Cannata Serio M, Rujano MA, Simons M (2018) Mutations in ATP6AP2 cause autophagic liver disease in humans. Autophagy 14:1088-1089. 
Castellucci VF, Kandel ER (1974) A quantal analysis of the synaptic depression underlying habituation of the gill-withdrawal reflex in Aplysia. Proc Natl Acad Sci USA 71:5004-5008.

Cattaert D, Birman S (2001) Blockade of the central generator of locomotor rhythm by noncompetitive NMDA receptor antagonists in Drosophila larvae. J Neurobiol 48:58-73.

Chintapalli VR, Wang J, Dow JA (2007) Using FlyAtlas to identify better Drosophila melanogaster models of human disease. Nat Genet 39:715-720.

Colacurcio DJ, Nixon RA (2016) Disorders of lysosomal acidification -the emerging role of $v$-ATPase in aging and neurodegenerative disease. Ageing Res Rev 32:75-88.

Collins MP, Forgac M (2020) Regulation and function of V-ATPases in physiology and disease. Biochim Biophys Acta 1862:183341.

Daniels RW, Collins CA, Chen K, Gelfand MV, Featherstone DE, DiAntonio A (2006) A single vesicular glutamate transporter is sufficient to fill a synaptic vesicle. Neuron 49:11-16.

Davie K, Janssens J, Koldere D, De Waegeneer M, Pech U, Kreft $Ł$, Aibar S, Makhzami S, Christiaens V, Bravo González-Blas C, Poovathingal S, Hulselmans G, Spanier KI, Moerman T, Vanspauwen B, Geurs S, Voet T, Lammertyn J, Thienpont B, et al. (2018) A single-cell transcriptome atlas of the aging Drosophila brain. Cell 174:982-998.

Davies SA, Goodwin SF, Kelly DC, Wang Z, Sözen MA, Kaiser K, Dow JA (1996) Analysis and inactivation of vha55, the gene encoding the vacuolar ATPase B-subunit in Drosophila melanogaster reveals a larval lethal phenotype. J Biol Chem 271:30677-30684.

Del Castillo J, Katz B (1954) Quantal components of the end-plate potential. J Physiol 124:560-573.

Dietzl G, Chen D, Schnorrer F, Su K-C, Barinova Y, Fellner M, Gasser B, Kinsey K, Oppel S, Scheiblauer S, Couto A, Marra V, Keleman K, Dickson BJ (2007) A genome-wide transgenic RNAi library for conditional gene inactivation in Drosophila. Nature 448:151-156.

Dubos A, Castells-Nobau A, Meziane H, Oortveld MAW, Houbaert X, lacono G, Martin C, Mittelhaeuser C, Lalanne V, Kramer JM, Bhukel A, Quentin C, Slabbert J, Verstreken P, Sigrist SJ, Messaddeq N, Birling MC, Selloum M, Stunnenberg HG, Humeau $Y$, et al. (2015) Conditional depletion of intellectual disability and Parkinsonism candidate gene ATP6AP2 in fly and mouse induces cognitive impairment and neurodegeneration. Hum Mol Genet 24:6736-6755.

Forgac M (2007) Vacuolar ATPases: rotary proton pumps in physiology and pathophysiology. Nat Rev Mol Cell Biol 8:917-929.

Goh GY, Huang H, Ullman J, Borre L, Hnasko TS, Trussell LO, Edwards RH (2011) Presynaptic regulation of quantal size: $\mathrm{K}+/ \mathrm{H}+$ exchange stimulates vesicular glutamate transport. Nat Neurosci 14:1285-1292.

Gowrisankaran S, Milosevic I (2020) Regulation of synaptic vesicle acidification at the neuronal synapse. IUBMB life 72:568-576.

Guida MC, Hermle T, Graham LA, Hauser V, Ryan M, Stevens TH, Simons M (2018) ATP6AP2 functions as a V-ATPase assembly factor in the endoplasmic reticulum. Mol Biol Cell 29:2156-2164.

Hazelrigg T, Levis R, Rubin GM (1984) Transformation of white locus DNA in Drosophila: dosage compensation, zeste interaction, and position effects. Cell 36:469-481.

Hiesinger PR, Fayyazuddin A, Mehta SQ, Rosenmund T, Schulze KL, Zhai RG, Verstreken P, Cao Y, Zhou Y, Kunz J, Bellen HJ (2005) The v-ATPase V0 subunit a1 is required for a late step in synaptic vesicle exocytosis in Drosophila. Cell 121:607-620.

Huang H, Trussell LO (2014) Presynaptic HCN channels regulate vesicular glutamate transport. Neuron 84:340-346.

Ichihara A, Yatabe MS (2019) The (pro) renin receptor in health and disease. Nat Rev Nephrol 15:693-712.

Inoue H, Noumi T, Nagata M, Murakami H, Kanazawa H (1999) Targeted disruption of the gene encoding the proteolipid subunit of mouse vacuolar $\mathrm{H}+$-ATPase leads to early embryonic lethality. Biochim Biophys Acta 1413:130-138.

Issa AR, Sun J, Petitgas C, Mesquita A, Dulac A, Robin M, Mollereau $B$, Jenny A, Chérif-Zahar B, Birman S (2018) The lysosomal membrane protein LAMP2A promotes autophagic flux and prevents
SNCA-induced Parkinson disease-like symptoms in the Drosophila brain. Autophagy 14:1898-1910.

Jan LY, Jan YN (1982) Antibodies to horseradish peroxidase as specific neuronal markers in Drosophila and in grasshopper embryos. Proc Natl Acad Sci USA 79:2700-2704.

Jansen EJ, Van Bakel NH, Coenen AJ, Van Dooren SH, Van Lith HA, Martens GJ (2010) An isoform of the vacuolar $(\mathrm{H}+)$-ATPase accessory subunit Ac45. Cell Mol Life Sci 67:629-640.

Jansen EJR, Martens GJM (2012) Novel insights into V-ATPase functioning: distinct roles for its accessory subunits ATP6AP1/Ac45 and ATP6AP2/(pro) renin receptor. Curr Protein Pept Sci 13:124133.

Jansen EJR, Timal S, Ryan M, Ashikov A, van Scherpenzeel M, Graham LA, Mandel H, Hoischen A, lancu TC, Raymond K, Steenbergen G, Gilissen C, Huijben K, van Bakel NHM, Maeda Y, Rodenburg RJ, Adamowicz M, Crushell E, Koenen H, Adams D, et al. (2016) ATP6AP1 deficiency causes an immunodeficiency with hepatopathy, cognitive impairment and abnormal protein glycosylation. Nat Commun 7:1-13.

Jansen EJR, van Bakel NHM, Benedict B, Olde Loohuis NFM, Hafmans TGM, Chim SM, Xu J, Kolk SM, Martens GJM (2021) Novel vertebrate-and brain-specific driver of neuronal outgrowth. Prog Neurobiol 202:102069.

Karunanithi S, Marin L, Wong K, Atwood HL (2002) Quantal size and variation determined by vesicle size in normal and mutant Drosophila glutamatergic synapses. J Neurosci 22:10267-10276.

Korvatska O, Strand NS, Berndt JD, Strovas T, Chen DH, Leverenz JB, Kiianitsa K, Mata IF, Karakoc E, Greenup JL, Bonkowski E, Chuang J, Moon RT, Eichler EE, Nickerson DA, Zabetian CP, Kraemer BC, Bird TD, Raskind WH (2013) Altered splicing of ATP6AP2 causes X-linked parkinsonism with spasticity (XPDS). Hum Mol Genet 22:3259-3268.

Kuno M (1971) Quantum aspects of central and ganglionic synaptic transmission in vertebrates. Physiol Rev 51:647-678.

Lee SK, Li W, Ryu SE, Rhim T, Ahnn J (2010) Vacuolar (H+)-ATPases in Caenorhabditis elegans: what can we learn about giant $\mathrm{H}+$ pumps from tiny worms? Biochim Biophys Acta 1797:1687-1695.

Lin DM, Goodman CS (1994) Ectopic and increased expression of fasciclin II alters motoneuron growth cone guidance. Neuron 13:507-523.

Mahr A, Aberle H (2006) The expression pattern of the Drosophila vesicular glutamate transporter: a marker protein for motoneurons and glutamatergic centers in the brain. Gene Expr Patterns 6:299309.

Manolson MF, Wu B, Proteau D, Taillon BE, Roberts BT, Hoyt MA, Jones EW (1994) STV1 gene encodes functional homologue of 95$\mathrm{kDa}$ yeast vacuolar $\mathrm{H}(+)-A T P a s e$ subunit Vph1p. J Biol Chem 269:14064-14074.

Maycox PR, Deckwerth T, Hell JW, Jahn R (1988) Glutamate uptake by brain synaptic vesicles. Energy dependence of transport and functional reconstitution in proteoliposomes. J Biol Chem 263:15423-15428.

Menon KP, Carrillo RA, Zinn K (2013) Development and plasticity of the Drosophila larval neuromuscular junction. Wiley Interdiscip Rev Dev Biol 2:647-670.

Miesenböck G, De Angelis DA, Rothman JE (1998) Visualizing secretion and synaptic transmission with $\mathrm{pH}$-sensitive green fluorescent proteins. Nature 394:192-195.

Mitchell AL, Attwood TK, Babbitt PC, Blum M, Bork P, Bridge A, Brown SD, Chang HY, El-Gebali S, Fraser MI, Gough J, Haft DR, Huang $\mathrm{H}$, Letunic I, Lopez R, Luciani A, Madeira F, Marchler-Bauer A, Mi H, Natale DA, et al. (2019) InterPro in 2019: improving coverage, classification and access to protein sequence annotations. Nucleic Acids Res 47:D351-D360.

Morel N, Gérard V, Shiff G (1998) Vacuolar H+-ATPase domains are transported separately in axons and assemble in Torpedo nerve endings. J Neurochem 71:1702-1708.

Morel N, Dedieu JC, Philippe JM (2003) Specific sorting of the a1 isoform of the V-H. + ATPase a subunit to nerve terminals where it 
associates with both synaptic vesicles and the presynaptic plasma membrane. J Cell Sci 116:4751-4762.

Moriyama Y, Maeda M, Futai M (1992) The role of V-ATPase in neuronal and endocrine systems. J Exp Biol 172:171-178.

Nagarkar-Jaiswal S, Lee PT, Campbell ME, Chen K, AnguianoZarate S, Cantu Gutierrez M, Busby T, Lin WW, He Y, Schulze KL, Booth BW, Evans-Holm M, Venken KJ, Levis RW, Spradling AC, Hoskins RA, Bellen HJ (2015) A library of MiMICs allows tagging of genes and reversible, spatial and temporal knockdown of proteins in Drosophila. Elife 4:e05338.

Nelson N (2003) A journey from mammals to yeast with vacuolar $\mathrm{H}+-$ ATPase (V-ATPase). J Bioenerg Biomembr 35:281-289.

Nguyen G (2010) The (pro) renin receptor in health and disease. Ann Med 42:13-18.

Parks AL, Cook KR, Belvin M, Dompe NA, Fawcett R, Huppert K, Tan LR, Winter CG, Bogart KP, Deal JE, Deal-Herr ME, Grant D, Marcinko M, Miyazaki WY, Robertson S, Shaw KJ, Tabios M, Vysotskaia V, Zhao L, Andrade RS, et al. (2004) Systematic generation of high-resolution deletion coverage of the Drosophila melanogaster genome. Nat Genet 36:288-292.

Perkins LA, Holderbaum L, Tao R, Hu Y, Sopko R, McCall K, YangZhou D, Flockhart I, Binari R, Shim HS, Miller A, Housden A, Foos M, Randkelv S, Kelley C, Namgyal P, Villalta C, Liu LP, Jiang X, Huan-Huan Q, et al. (2015) The transgenic RNAi project at Harvard Medical School: resources and validation. Genetics 201:843-852.

Port F, Chen HM, Lee T, Bullock SL (2014) Optimized CRISPR/Cas tools for efficient germline and somatic genome engineering in Drosophila. Proc Natl Acad Sci USA 111:E2967-E2976.

Poskanzer KE, Davis GW (2004) Mobilization and fusion of a non-recycling pool of synaptic vesicles under conditions of endocytic blockade. Neuropharmacology 47:714-723.

Rath A, Glibowicka M, Nadeau VG, Chen G, Deber CM (2009) Detergent binding explains anomalous SDS-PAGE migration of membrane proteins. Proc Natl Acad Sci USA 106:1760-1765.

Risse B, Otto N, Berh D, Jiang X, Klämbt C (2014) FIM imaging and FIMtrack: two new tools allowing high-throughput and cost effective locomotion analysis. J Vis Exp (94):e52207.

Rujano MA, Cannata Serio M, Panasyuk G, Péanne R, Reunert J, Rymen D, Hauser V, Park JH, Freisinger P, Souche E, Guida MC, Maier EM, Wada Y, Jäger S, Krogan NJ, Kretz O, Nobre S, Garcia P, Quelhas D, Bird TD, et al. (2017) Mutations in the X-linked ATP6AP2 cause a glycosylation disorder with autophagic defects. J Exp Med 214:3707-3729.
Saroussi S, Nelson N (2009) The little we know on the structure and machinery of V-ATPase. J Exp Biol 212:1604-1610.

Sautin YY, Lu M, Gaugler A, Zhang L, Gluck SL (2005) Phosphatidylinositol 3-kinase-mediated effects of glucose on vacuolar $\mathrm{H}+-$-ATPase assembly, translocation, and acidification of intracellular compartments in renal epithelial cells. Mol Cell Biol 25:575-589.

Schoonderwoert VTG, Martens GJ (2002a) Structural gene organization and evolutionary aspects of the V-ATPase accessory subunit Ac45. Biochim Biophys Acta 1574:245-254.

Schoonderwoert VTG, Martens GJ (2002b) Targeted disruption of the mouse gene encoding the V-ATPase accessory subunit Ac45. Mol Membr Biol 19:67-71.

Stewart BA, Atwood HL, Renger JJ, Wang J, Wu CF (1994) Improved stability of Drosophila larval neuromuscular preparations in haemolymph-like physiological solutions. J Comp Physiol A Neuroethol Sens Neural Behav Physiol 175:179-191.

Sun-Wada GH, Murata Y, Yamamoto A, Kanazawa H, Wada Y, Futai $M(2000)$ Acidic endomembrane organelles are required for mouse postimplantation development. Dev Biol 228:315-325.

Tabb JS, Kish PE, Van Dyke R, Ueda T (1992) Glutamate transport into synaptic vesicles. Roles of membrane potential, $\mathrm{pH}$ gradient, and intravesicular pH. J Biol Chem 267:15412-15418.

Takamori S (2016) Presynaptic molecular determinants of quantal size. Front Synaptic Neurosci 8:2.

Thurmond J, Goodman JL, Strelets VB, Attrill H, Gramates LS, Marygold SJ, Matthews BB, Millburn G, Antonazzo G, Trovisco V, Kaufman TC, Calvi BR; FlyBase Consortium (2019) FlyBase 2.0: the next generation. Nucleic Acids Res 47:D759-D765.

Vasanthakumar T, Rubinstein JL (2020) Structure and Roles of Vtype ATPases. Trends Biochem Sci 45:295-307.

Verstreken P, Ohyama T, Bellen HJ (2008) FM 1-43 labeling of synaptic vesicle pools at the Drosophila neuromuscular junction. Methods Mol Biol 440:349-369.

Voss M, Vitavska O, Walz B, Wieczorek H, Baumann O (2007) Stimulus-induced phosphorylation of vacuolar $\mathrm{H}+$-ATPase by protein kinase A. J Biol Chem 282:33735-33742.

Wu TH, Lu YN, Chuang CL, Wu CL, Chiang AS, Krantz DE, Chang HY (2013) Loss of vesicular dopamine release precedes tauopathy in degenerative dopaminergic neurons in a Drosophila model expressing human tau. Acta Neuropathol 125:711-725. 